\title{
Spatial Modulation Aided Zero-Padded Single Carrier Transmission for Dispersive Channels
}

\author{
Rakshith Rajashekar, Student Member, IEEE, K.V.S. Hari, Senior Member, IEEE, and L. Hanzo, Fellow, IEEE
}

\begin{abstract}
In this paper, we consider spatial modulation (SM) operating in a frequency-selective single-carrier (SC) communication scenario and propose zero-padding instead of the cyclicprefix considered in the existing literature. We show that the zeropadded single-carrier (ZP-SC) SM system offers full multipath diversity under maximum-likelihood (ML) detection, unlike the cyclic-prefix based SM system. Furthermore, we show that the order of ML detection complexity in our proposed ZP-SC SM system is independent of the frame length and depends only on the number of multipath links between the transmitter and the receiver. Thus, we show that the zero-padding applied in the SC SM system has two advantages over the cyclic prefix: 1) achieves full multipath diversity, and 2) imposes a relatively low ML detection complexity. Furthermore, we extend the partial interference cancellation receiver (PIC-R) proposed by Guo and Xia for the detection of space-time block codes (STBCs) in order to convert the ZP-SC system into a set of narrowband subsystems experiencing flat-fading. We show that full rank STBC transmissions over these subsystems achieves full transmit, receive as well as multipath diversity for the PIC-R. Furthermore, we show that the ZP-SC SM system achieves receive and multipath diversity for the PIC-R at a detection complexity order which is the same as that of the SM system in flat-fading scenario. Our simulation results demonstrate that the symbol error ratio performance of the proposed linear receiver for the ZP-SC SM system is significantly better than that of the SM in cyclic prefix based orthogonal frequency division multiplexing as well as of the SM in the cyclic-prefixed and zero-padded single carrier systems relying on zero-forcing/minimum mean-squared error equalizer based receivers.
\end{abstract}

Index Terms-Spatial modulation, zero padded single carrier communication, partial interference cancellation, diversity, decoding complexity.

\section{INTRODUCTION}

$\mathbf{T}$ $\mathrm{HE}$ benefits of multi-antenna aided transmission schemes are achieved at the cost of a substantial decoding complexity imposed at the receiver. A substantial contributor to the high decoding complexity at the receiver is the interantenna interference (IAI) which arises due to the simultaneous activation of all the antennas at the transmitter. For example, in the vertical Bell laboratories layered space-time

Manuscript received January 5, 2013; revised March 11, 2013. The editor coordinating the review of this paper and approving it for publication was E. $\mathrm{Au}$.

R. Rajashekar and K.V.S. Hari are with the Department of Electrical Communication Engineering, Indian Institute of Science, India (e-mail: \{rakshithmr, hari\}@ece.iisc.ernet.in).

L. Hanzo is with the School of ECS, University of Southampton, UK (email: lh@ecs.soton.ac.uk).

The financial support of the DST, India, and of the EPSRC, UK under the auspices of the India-UK Advanced Technology Center (IU-ATC) is gratefully acknowledged. L. Hanzo would also like to thank the ERC for their support under the Advanced Fellow Grant.

Digital Object Identifier 10.1109/TCOMM.2013.043013.130011

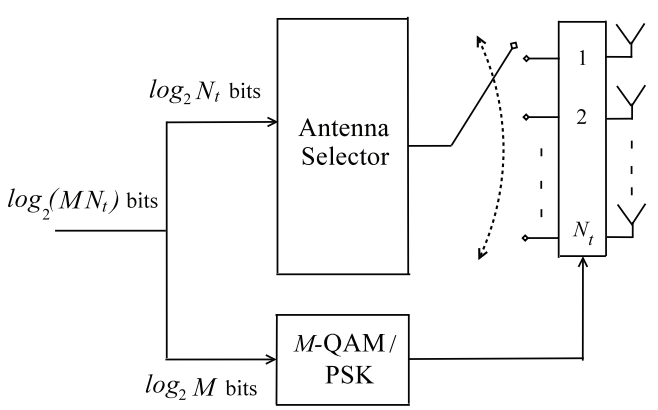

Fig. 1. Pictorial representation of the spatial modulation system.

(V-BLAST) architecture [1], the maximum likelihood (ML) decoding complexity is exponential in the number of transmit antennas. Although the sphere decoding (SD) algorithm associated with a sufficiently high search-radius guarantees the ML performance [2], [3], the reduction in search complexity due to SD at low signal-to-noise ratios (SNR) remains modest compared to that at high SNRs.

Spatial modulation (SM) [4]-[6] is a recently developed low-complexity multiple-input multiple-output (MIMO) communication scheme that relies on a single radio frequency (RF) chain at the transmitter. In this scheme, the information bitstream is divided into blocks of $\log _{2}\left(N_{t} M\right)$ bits, and in each block, $\log _{2}(M)$ bits select a symbol $s$ from an $M$-ary signal set (such as $M$-QAM or -PSK), and $\log _{2}\left(N_{t}\right)$ bits select an antenna out of $N_{t}$ transmit antennas for the transmission of the symbol $s$. Fig. 1 gives a pictorial representation of the SM scheme. Since only one transmit antenna is activated in any symbol duration, the IAI is completely avoided at the receiver, thereby resulting in a low-complexity single-stream ML detector [7], [8] at the receiver.

Below we provide a brief overview of the recent contributions on SM. Several reduced-complexity detectors were proposed for SM in [9]-[12] that achieve near-ML performance. Furthermore, the robustness of the SM system to channel imperfections was studied in comparison to conventional MIMO (C-MIMO) systems in [13], [14]. Transmit diversity techniques were conceived for SM systems in [15]-[18], while power allocation algorithms were studied in [19], [20]. A detailed study of the SM system employing adaptive modulation and transmit antenna selection relying on feedback information obtained from the receiver can be found in [21]-[23]. In the SM scheme studied in the existing literature [4]-[23], the channel has routinely been assumed to be a frequency-flat block Rayleigh fading. However, very recently, the SM scheme was studied in a frequency selective channel using a cyclic- 
prefixed (CP) single carrier (SC) communication system [24]. Additional related studies include that of [25]. Following are the important inferences drawn in [24]:

- The SM scheme in CP-SC communication cannot exploit multipath diversity (with ML decoding at the receiver), hence the diversity order achieved is only $N_{r}$, where $N_{r}$ is the number of receive antennas.

- If $K$ is the number of SM symbols in a data-frame, then the order of ML decoding complexity is given by $\left(N_{t} M\right)^{K}$, i.e. the order of ML decoding complexity is exponential in $K$.

Against this background, following are the contributions of this paper:

1) Precoding the sequence of SM symbols by the inverse fast Fourier transform (IFFT) matrix in addition to cyclic prefixing yields parallel channels leading to reduced complexity detection, but this requires $N_{t} \mathrm{RF}$ chains at the transmitter. However, adding $\mathrm{CP}$ alone will not provide any detection advantage in the SM system having a single RF chain at the transmitter. Instead of cyclic prefixing, we propose zero-padding (ZP) and show that the SM scheme employed for ZP$\mathrm{SC}$ communication offers full multipath diversity [see Proposition 1, Section II], while requiring only one RF chain at the transmitter.

2) Furthermore, we show that the order of ML detection complexity in the ZP-SC SM system is $\left(N_{t} M\right)^{P}$, where $P$ is the number of taps in the multipath channel, unlike that of the CP-SC SM scheme, where the ML detection complexity is of the order $\left(N_{t} M\right)^{K}$ [see Proposition 2, Section III]. This is achieved by formulating the ZPSC SM system as a space-time block code (STBC), and then suitably tailoring and applying the generalized distributive law (GDL) conceived for ML detection of STBCs [26].

3) We extend the partial interference-cancellation based receiver (PIC-R) [27], [28] to a ZP-SC system and show that the resultant ZP-SC system can be converted into a set of block fading subsystems. Furthermore, we demonstrate that the transmission of any full-rank STBC over these subsystems offers full transmit, receive and multipath diversity under PIC-R [see Proposition 3, Section IV $]^{1}$. Finally, we show that the SM applied in the ZP-SC system achieves both multipath and receive diversity under PIC-R at a decoding complexity order, which is the same as that of the SM system operating in a flat-fading scenario. Furthermore, by exploiting the displacement structure [31]-[33] of the block Toeplitz matrices, we propose a reduced complexity successive interference cancellation based PIC-R, whose complex-

\footnotetext{
${ }^{1}$ The PIC-R [27] was mainly designed for low-complexity decoding of STBCs, while exploiting the full transmit diversity potential of the code. Note that no other STBCs are capable of offering a full-diversity potential using PIC-R, but only those that satisfy the requirements posed by Theorem 1 of [27]. Several STBCs were designed that offer full-diversity with the aid of PIC-R (e.g. [29], [30]), which were all considered for a flat-fading scenario. In contrast to this, we extend the PIC-R for a zero-padded MIMO system operating in a dispersive channel, which has not been done in the existing literature. Since the focus of the paper is on SM systems, we restrict our study to the SM system itself and do not dwell on the STBCs.
}

ity order is the same as that of the conventional zeroforcing (ZF)/minimum mean-squared error (MMSE) equalizer based receiver.

A detailed study of the ZP-SC C-MIMO system can be found in [34]-[37]. Table I compares various properties of the SM system to those of the C-MIMO and SIMO systems, when operating in a dispersive channel with the aid of ZP. It is evident from Table I that the SM system achieves the same diversity order as that of the SIMO system and achieves a $\log _{2} N_{t}$ bits per channel use (bpcu) higher throughput, while still requiring only one RF chain at the transmitter. Our objective in this paper is to study the performance improvement due to the additional antennas used by the SM system over the SIMO system and further compare it with the performance of the C-MIMO system which needs multiple RF chains at the transmitter.

Notations: Lowercase boldface letters represent vectors. Uppercase boldface letters denote matrices, while $\operatorname{Tr}(\cdot)$ is the trace of a matrix. Furthermore, $\|\cdot\|$ represents the twonorm of a vector, or the Frobenious norm of a matrix. The notations of $(\cdot)^{T}$ and $(\cdot)^{H}$ indicate the transpose and Hermitian transpose of a vector/matrix, respectively, while $|\cdot|$ represents the cardinality of a given set, or the magnitude of a complex quantity. $\mathbf{H}([a: b],:)$ represents a matrix with rows $a, a+1, \ldots, b-1, b$ of $\mathbf{H}$ and $\mathbf{H}(:,[a: b])$ is a matrix with columns $a, a+1, \ldots, b-1, b$ of $\mathbf{H}$. $\mathbb{R}$ and $\mathbb{C}$ represent the field of real and complex numbers, respectively, while $\mathcal{C N}\left(\mu, \sigma^{2}\right)$ denotes a complex Gaussian random variable with mean $\mu$ and variance $\sigma^{2}$. If $S_{1}$ and $S_{2}$ are two sets such that $S_{2} \subset S_{1}$, then $X=S_{1} \backslash S_{2}$ represents a set containing all the elements of $S_{1}$ that are not present in $S_{2} . S_{3}=S_{1} \times S_{2}$ represents the Cartesian product of the sets $S_{1}$ and $S_{2}$.

\section{ZP-SC SM SYSTEM}

We consider a MIMO system having $N_{t}$ transmit as well as $N_{r}$ receive antennas and a quasi-static, frequency-selective fading channel having $P$ resolvable multipath links between each transmit and receive antenna pairs. The received vector during the $i^{\text {th }}$ channel use is given by

$$
\mathbf{y}_{i}=\sum_{j=0}^{P-1} \mathbf{H}_{j} \mathbf{x}_{i-j}+\mathbf{n}_{i},
$$

where $\mathbf{x}_{k} \in \mathbb{C}^{N_{t} \times 1}$ and $\mathbf{y}_{k} \in \mathbb{C}^{N_{r} \times 1}$ are the transmitted and received vectors in the $k^{\text {th }}$ channel use, $\mathbf{H}_{j} \in \mathbb{C}^{N_{r} \times N_{t}}$ is the $j^{\text {th }}$ multipath channel matrix, and $\mathbf{n}_{k} \in \mathbb{C}^{N_{r} \times 1}$ is the noise vector in the $k^{\text {th }}$ channel use. The entries of the multipath channel matrices and the noise vector are from $\mathcal{C N}(0,1)$ and $\mathcal{C N}\left(0, \sigma^{2}\right)$, respectively, where $\sigma^{2}$ is the noise variance per complex dimension. We assume that $\mathbf{x}_{k}$ has unit energy and take $\sigma^{2}=\frac{1}{\rho}$ in order to ensure that the average received SNR at each receive antenna is $\rho$.

Assuming that $K$ channel uses/transmission symbols constituting a single data frame and each data frame is prefixed with $(P-1)$ zeros $^{2}$, we have $q^{\text {th }}$ zero-padded $N_{t} \times(K+P-1)$ -

\footnotetext{
${ }^{2}$ Prefixing a data frame with $(P-1)$ zeros is equivalent to the transmitter being silent for $(P-1)$ channel uses before commencing the transmission of the data frame.
} 
TABLE I

COMPARISON OF THE SM, C-MIMO AND SIMO SYSTEMS OPERATING IN A DISPERSIVE CHANNEL WITH THE AID OF ZP.

\begin{tabular}{|l||c|c|c|}
\hline & ZP-SC SM & ZP-SC C-MIMO & ZP-SC SIMO \\
\hline \hline $\begin{array}{l}\text { No. of transmit } \\
\text { RF chains required }\end{array}$ & 1 & $N_{t}$ & 1 \\
\hline $\begin{array}{l}\text { Throughput } \\
\text { (bpcu) }\end{array}$ & $\log _{2}\left(N_{t} M\right)$ & $N_{t} \log _{2}(M)$ & $\log _{2}(M)$ \\
\hline $\begin{array}{l}\text { Rate } \\
\text { (cpcu) }\end{array}$ & 1 & $N_{t}$ & 1 \\
\hline $\begin{array}{l}\text { ML decoding } \\
\text { complexity } \\
\text { order }\end{array}$ & $\begin{array}{c}\left(N_{t} M\right)^{P} \\
(\text { Proposition 2) }\end{array}$ & $\begin{array}{c}M^{\left(N_{t} P\right)} \\
(\text { Chapter 5, [46]) }\end{array}$ & $M^{P}$ \\
\hline $\begin{array}{l}\text { Achievable } \\
\text { diversity } \\
\text { order }\end{array}$ & $\begin{array}{c}P N_{r} \text { with } \\
\text { PIC-R/PIC-R-SIC } \\
\text { (Corollary 1) }\end{array}$ & $\begin{array}{c}P N_{r}-N_{t}+1 \\
\text { with ZF/MMSE } \\
(\text { Proposition 1, [37]) }\end{array}$ & $\begin{array}{c}P N_{r} \\
\text { with ZF/MMSE }\end{array}$ \\
\hline
\end{tabular}

bpcu : bits per channel use

cpcu : complex symbols per channel use

element data frame given by

$$
[\underbrace{\mathbf{0 , 0}, \ldots, \mathbf{0}}_{P-1 \text { zero vectors }}, \underbrace{\mathbf{x}_{K(q-1)+1}, \mathbf{x}_{K(q-1)+2}, \ldots, \mathbf{x}_{K(q-1)+K}}_{K \text { data vectors }}],
$$

where $\mathbf{0}$ denotes an all-zero vector of size $N_{t} \times 1$. The $q^{\text {th }}$ received data frame is given by (3), where $\mathbf{O}$ represents a $\left(N_{r} \times N_{t}\right)$-element null matrix and $K^{\prime}=K+P-1$. Since the number of multipaths is $P,(P-1)$ length zero-padding ensures that the successive data frames do not suffer from inter frame interference. In the SM scheme, each transmitted vector is of the form [4]

$$
\mathbf{x}_{k}=[\underbrace{0, \ldots, 0}_{l_{k}-1}, s_{k}, \underbrace{0, \ldots, 0}_{N_{t}-l_{k}}]^{T} \in \mathbb{C}^{N_{t} \times 1},
$$

where $s_{k}$ is a complex symbol from the signal set $S$ having $|S|=M$ and $l_{k} \in L=\{i\}_{i=1}^{N_{t}}$. Thus, each $\mathbf{x}_{k}$ may assume $N_{t} M$ different values.

\section{A. ML Detection and Multipath Diversity}

From (3), it becomes clear that $\hat{\mathbf{x}}$ may assume $\left(N_{t} M\right)^{K}$ different values, and hence the ML solution is given by

$$
\hat{\mathbf{x}}_{M L}=\arg \min _{\hat{\mathbf{x}} \in \mathcal{X}}\|\hat{\mathbf{y}}-\hat{\mathbf{H}} \hat{\mathbf{x}}\|_{2}^{2}
$$

where $\mathcal{X}$ is the set of all legitimate transmit vectors with $|\mathcal{X}|=$ $\left(N_{t} M\right)^{K}$. For the ease of presentation, we assume $N_{r}=1$ and consider only the first frame in our analysis, but all the results presented hold for arbitrary $N_{r}$. Then, the first data frame received is given by

$$
\hat{\mathbf{y}}=\hat{\mathbf{H}} \hat{\mathbf{x}}+\hat{\mathbf{n}},
$$

where $\hat{\mathbf{y}}=\left[y_{1}, y_{2}, \ldots, y_{K+P-1}\right]^{T}$,

$$
\hat{\mathbf{H}}=\left[\begin{array}{ccccc}
\mathbf{h}_{0} & \mathbf{0} & \cdots & \mathbf{0} & \mathbf{0} \\
\mathbf{h}_{1} & \mathbf{h}_{0} & \cdots & \mathbf{0} & \mathbf{0} \\
\vdots & \vdots & \ddots & \vdots & \vdots \\
\mathbf{h}_{P-1} & \mathbf{h}_{P-2} & \ddots & \mathbf{h}_{0} & \mathbf{0} \\
\mathbf{0} & \mathbf{h}_{P-1} & \ddots & \mathbf{h}_{1} & \mathbf{h}_{0} \\
\vdots & \vdots & \ddots & \vdots & \vdots \\
\mathbf{0} & \mathbf{0} & \cdots & \mathbf{h}_{P-1} & \mathbf{h}_{P-2} \\
\mathbf{0} & \mathbf{0} & \cdots & \mathbf{0} & \mathbf{h}_{P-1}
\end{array}\right]_{(K+P-1) \times K N_{t}}
$$

so that $\mathbf{h}_{j} \in \mathbb{C}^{1 \times N_{t}}$ corresponds to the $j^{\text {th }}$ set of multipath links from $N_{t}$ transmit antennas to the receive antenna, $\mathbf{0}$ is a $\left(1 \times N_{t}\right)$-element null vector, $\hat{\mathbf{n}}=\left[n_{1}, n_{2}, \ldots, n_{K+P-1}\right]$ and $\hat{\mathbf{x}}=\left[\mathbf{x}_{1}^{T}, \mathbf{x}_{2}^{T}, \ldots, \mathbf{x}_{K}^{T}\right]^{T}$.

Let

$$
\mathbf{A}_{i}=\left[\mathbf{e}_{P(i-1)+1}, \mathbf{e}_{P(i-1)+2}, \ldots, \mathbf{e}_{P(i-1)+P}, \mathbf{O}_{N_{t} P \times(K-1)}\right]
$$

for $1 \leq i \leq N_{t}$, where $\left\{\mathbf{e}_{k}\right\}_{k=1}^{N_{t} P}$ is the set of natural basis for $\mathbb{R}^{N_{t} P}, \mathcal{B}=\left\{\mathbf{A}_{1}, \mathbf{A}_{2}, \ldots, \mathbf{A}_{N_{t}}\right\}$ and

$$
\mathbf{T}=\left[\begin{array}{cccccc}
0 & 1 & 0 & 0 & \ldots & 0 \\
0 & 0 & 1 & 0 & \ldots & 0 \\
0 & 0 & 0 & 1 & \ldots & 0 \\
\vdots & \vdots & \vdots & \vdots & \ddots & \vdots \\
0 & 0 & 0 & 0 & \ldots & 1 \\
1 & 0 & 0 & 0 & \ldots & 0
\end{array}\right] \in \mathbb{R}^{(K+P-1) \times(K+P-1)}
$$

be the shift-operator that circularly shifts the columns of the matrix that it operates on, i.e. we have

$\left[\mathbf{p}_{1}, \mathbf{p}_{2}, \ldots, \mathbf{p}_{K+P-1}\right] \mathbf{T}=\left[\mathbf{p}_{K+P-1}, \mathbf{p}_{1}, \mathbf{p}_{2}, \ldots, \mathbf{p}_{K+P-2}\right]$.

The system in (5) may be equivalently written as

$$
\hat{\mathbf{y}}^{T}=\overline{\mathbf{H}} \mathbf{X}+\hat{\mathbf{n}}^{T},
$$

where $\overline{\mathbf{H}}=\left[\overline{\mathbf{h}}_{1}, \overline{\mathbf{h}}_{2}, \ldots, \overline{\mathbf{h}}_{N_{t}}\right] \in \mathbb{C}^{1 \times N_{t} P}$, and $\overline{\mathbf{h}}_{i}=$ $\left[\mathbf{h}_{0}(i), \mathbf{h}_{1}(i), \ldots, \mathbf{h}_{P-1}(i)\right]$ for $1 \leq i \leq N_{t}$ and $\mathbf{X} \in$ 


$$
\underbrace{\left[\begin{array}{c}
\mathbf{y}_{K^{\prime}(q-1)+1} \\
\mathbf{y}_{K^{\prime}(q-1)+2} \\
\vdots \\
\mathbf{y}_{K^{\prime}(q-1)+K^{\prime}}
\end{array}\right]}_{\hat{\mathbf{y}}}=\underbrace{\left[\begin{array}{ccccc}
\mathbf{H}_{0} & \mathbf{O} & \ldots & \mathbf{O} & \mathbf{O} \\
\mathbf{H}_{1} & \mathbf{H}_{0} & \ldots & \mathbf{O} & \mathbf{O} \\
\vdots & \vdots & \ddots & \vdots & \vdots \\
\mathbf{H}_{P-1} & \mathbf{H}_{P-2} & \ddots & \mathbf{H}_{0} & \mathbf{O} \\
\mathbf{O} & \mathbf{H}_{P-1} & \ddots & \mathbf{H}_{1} & \mathbf{H}_{0} \\
\vdots & \vdots & \ddots & \vdots & \vdots \\
\mathbf{O} & \mathbf{O} & \ldots & \mathbf{H}_{P-1} & \mathbf{H}_{P-2} \\
\mathbf{O} & \mathbf{O} & \ldots & \mathbf{O} & \mathbf{H}_{P-1}
\end{array}\right]}_{\hat{\mathbf{n}}} \underbrace{\left[\begin{array}{c}
\mathbf{x}_{K(q-1)+1} \\
\mathbf{x}_{K(q-1)+2} \\
\vdots \\
\mathbf{x}_{K(q-1)+K}
\end{array}\right]}_{\hat{\mathbf{x}}} \underbrace{\left[\begin{array}{c}
\mathbf{n}_{K^{\prime}(q-1)+1} \\
\mathbf{n}_{K^{\prime}(q-1)+2} \\
\vdots \\
\mathbf{n}_{K^{\prime}(q-1)+K^{\prime}}
\end{array}\right]}_{\hat{\mathbf{H}}_{\text {of size } N_{r}(K+P-1) \times K N_{t}}}
$$

$\mathbb{C}^{N_{t} P \times(K+P-1)}$ is from a finite set of matrices $\mathcal{C}$, given by

$$
\mathcal{C}=\left\{\sum_{k=1}^{K} s_{k} \mathbf{B}_{k} \mathbf{T}^{k-1} \mid s_{k} \in S, \mathbf{B}_{k} \in \mathcal{B} \text { for } 1 \leq k \leq K\right\},
$$

where $\mathbf{T}^{0}$ is taken as $\mathbf{I}_{K+P-1}$. It may be readily seen from (11) that $|\mathcal{C}|=|S|^{K}|\mathcal{B}|^{K}=\left(M N_{t}\right)^{K}$. From (10) and (11), it becomes clear that the ZP-SC SM scheme can be interpreted as an STBC.

Following example illustrates the equivalence between the system models of (5) and (10).

Example 1: Consider a system having $N_{t}=2, N_{r}=1$, $P=3$ and $K=3$. Let $\hat{\mathbf{x}}=\left[s_{1}, 0,0, s_{2}, 0, s_{3}\right]^{T}$ and $\hat{\mathbf{h}}_{i}=$ $\left[\mathbf{h}_{i}(1), \mathbf{h}_{i}(2)\right]$ for $i=1,2,3$. Then from (5) we have

$\hat{\mathbf{H}} \hat{\mathbf{x}}=\left[\begin{array}{cccccc}\mathbf{h}_{0}(1) & \mathbf{h}_{0}(2) & 0 & 0 & 0 & 0 \\ \mathbf{h}_{1}(1) & \mathbf{h}_{1}(2) & \mathbf{h}_{0}(1) & \mathbf{h}_{0}(2) & 0 & 0 \\ \mathbf{h}_{2}(1) & \mathbf{h}_{2}(2) & \mathbf{h}_{1}(1) & \mathbf{h}_{1}(2) & \mathbf{h}_{0}(1) & \mathbf{h}_{0}(2) \\ 0 & 0 & \mathbf{h}_{2}(1) & \mathbf{h}_{2}(2) & \mathbf{h}_{1}(1) & \mathbf{h}_{1}(2) \\ 0 & 0 & 0 & 0 & \mathbf{h}_{2}(1) & \mathbf{h}_{2}(2)\end{array}\right]\left[\begin{array}{c}s_{1} \\ 0 \\ 0 \\ s_{2} \\ 0 \\ s_{3}\end{array}\right]$,

whose transpose is equivalent to

$$
\begin{aligned}
(\hat{\mathbf{H}} \hat{\mathbf{x}})^{T}=\overline{\mathbf{H}} \mathbf{X}=\left[\mathbf{h}_{0}(1), \mathbf{h}_{1}(1), \mathbf{h}_{2}(1), \mathbf{h}_{0}(2), \mathbf{h}_{1}(2), \mathbf{h}_{2}(2)\right] \times \\
\qquad\left[\begin{array}{cccccc}
s_{1} & 0 & 0 & 0 & 0 \\
0 & s_{1} & 0 & 0 & 0 \\
0 & 0 & s_{1} & 0 & 0 \\
0 & s_{2} & s_{3} & 0 & 0 \\
0 & 0 & s_{2} & s_{3} & 0 \\
0 & 0 & 0 & s_{2} & s_{3}
\end{array}\right] .
\end{aligned}
$$

Proposition 1: In a multipath channel having $P$ resolvable links between each of the transmit antennas and the receive antenna pairs, the ZP-SC SM scheme can achieve a diversity order of $P$ in conjunction with ML detection.

Proof: Proof is provided in Appendix A.

It can be readily shown that with $N_{r}$ receive antennas the ZP-SC SM scheme achieves a diversity order of $N_{r} P$.

Remark 1: If $\mathcal{B}$ is a singleton set, say $\mathcal{B}=\left\{\mathbf{A}_{1}\right\}$, then the ZP-SC SM system reduces to a ZP$\mathrm{SC}$ SIMO system, in which case we have $\mathcal{C}=\left\{\sum_{k=1}^{K} s_{k} \mathbf{A}_{1} \mathbf{T}^{k-1} \mid s_{k} \in S\right.$ for $\left.1 \leq k \leq K\right\}$. It can be readily seen that the elements of the set $\mathcal{C}$ in this case are Toeplitz matrices and hence $\mathcal{C}$ is equivalent to the Toeplitz STBC [38], which is known to provide full transmit diversity.

\section{PRoposed LOW-COMPLEXITY GDL-BASED ML DETECTION FOR THE ZP-SC SM SYSTEM}

In this section, we show that the ML decoding complexity order in the ZP-SC SM system can be reduced to $\left(N_{t} M\right)^{P}$ with the aid of the GDL. For a review of GDL, the reader is referred to [26]. The main idea in achieving a reducedcomplexity ML detection is by exploiting the fact that the transmitted SM symbols suffer interference from only $P-1$ previously transmitted symbols, regardless of the length of the data frame.

\section{A. Reduced complexity GDL-based ML detection in ZP-SC SM system}

In this section we will show that the order of ML decoding complexity in the ZP-SC SM system may be reduced from $\left(N_{t} M\right)^{K}$ to $\left(N_{t} M\right)^{P}$ with the aid of GDL.

Considering the STBC given in (11), for a fixed $\left\{\mathbf{B}_{k}\right\}_{k=1}^{K}=$ $\left\{\mathbf{A}_{l_{k}}\right\}_{k=1}^{K}$, where $l_{k} \in L=\{i\}_{i=1}^{N_{t}}$, we may have $\mathbf{x}_{n}=$ $\left[s_{n_{I}}, s_{n_{Q}}\right]$ for $1 \leq n \leq K$ as the set of encoding groups, where $s_{n_{I}}=\Re\left(s_{n}\right)$ and $s_{n_{Q}}=\Im\left(s_{n}\right)$. Since the transmitted symbol as well as the activated transmit antenna together convey information in the SM scheme, we extend the set of encoding groups by additionally including the antenna index associated with the transmitted symbol, yielding $\mathbf{x}_{n}^{\prime}=$ $\left[s_{n_{I}}, s_{n_{Q}} ; l_{n}\right] \in \mathcal{A}_{n}^{\prime}=S \times L$ for $1 \leq n \leq K$, and hence refer to them as extended encoding groups. Furthermore, let $s_{2 i-1}=s_{i_{I}}, s_{2 i}=s_{i_{Q}}, \mathbf{M}_{2 i-1}=\mathbf{M}_{2 i-1}\left(l_{2 i-1}\right)=\mathbf{A}_{l_{i}} \mathbf{T}^{i-1}$ and $\mathbf{M}_{2 i}=\mathbf{M}_{2 i}\left(l_{i}\right)=j \mathbf{A}_{l_{i}} \mathbf{T}^{i-1}$ for $1 \leq i \leq K$, where $\mathbf{M}_{k}\left(l_{j}\right)$ indicates that $\mathbf{M}_{k}$ depends on $l_{j}$. Then, the local kernel associated with $\mathbf{x}_{n}^{\prime}$ is given by $\alpha_{n}^{\prime}\left(\mathbf{x}_{n}^{\prime}\right)=$ $\sum_{i \in \psi_{n}}\left[s_{i} \zeta_{i}\left(l_{n}\right)+s_{i}^{2} \zeta_{i, i}\left(l_{n}\right)\right]+\sum_{\substack{j>j \\ i, j \in \psi_{n}}} s_{i} s_{j} \zeta_{i, j}\left(l_{n}\right)$, where $\psi_{n}=\{2 n-1,2 n\}$, while that associated with $\left(\mathbf{x}_{n}^{\prime}, \mathbf{x}_{m}^{\prime}\right)$ is given by $\alpha_{n, m}^{\prime}\left(\mathbf{x}_{n}^{\prime}, \mathbf{x}_{m}^{\prime}\right)=\sum_{\substack{i \in \psi_{n} \\ j \in \psi_{m}}} s_{i} s_{j} \zeta_{i, j}\left(l_{n}, l_{m}\right)$. The directed message emanating from vertex $u$ to $v$ with extended encoding groups is given by the range of the function $\mu_{u, v}^{\prime}: \mathcal{A}_{\mathcal{I}_{u} \cap \mathcal{I}_{v}}^{\prime} \rightarrow \mathbb{R}, \mu_{u, v}^{\prime}\left(\mathbf{x}_{\mathcal{I}_{u} \cap \mathcal{I}_{v}}^{\prime}\right)=$ $\min _{\mathbf{x}_{\mathcal{I}_{u} \backslash \mathcal{I}_{v}}^{\prime}}\left(\alpha_{u}^{\prime}\left(\mathbf{x}_{\mathcal{I}_{u}}^{\prime}\right)+\sum_{w a d j u} u \mu_{w, u}\left(\mathbf{x}_{\mathcal{I}_{w} \cap \mathcal{I}_{u}}^{\prime}\right)\right)$.

Definition 1: Two extended encoding groups $\mathbf{x}_{n}^{\prime}$ and $\mathbf{x}_{m}^{\prime}$ are said to be non-interfering if $\left(\mathbf{A}_{l_{n}} \mathbf{T}^{n-1}\right)\left(\mathbf{A}_{l_{m}} \mathbf{T}^{m-1}\right)^{H}+$ $\left(\mathbf{A}_{l_{m}} \mathbf{T}^{m-1}\right)\left(\mathbf{A}_{l_{n}} \mathbf{T}^{n-1}\right)^{H}=\mathbf{O}$ for all $1 \leq l_{n} \leq N_{t}$ and $1 \leq l_{m} \leq N_{t}$.

Note that the above definition ensures that, for any given $l_{n}$ and $l_{m}$ in $L, \nexists s \in\left[s_{n_{I}}, s_{n_{Q}}\right]$ and $s^{\prime} \in\left[s_{m_{I}}, s_{m_{Q}}\right]$ such that their weight matrices are not Hurwitz-Radon orthogonal. Since the weight matrices associated with the symbols $s_{n_{I}}$ and $s_{n_{Q}}$ are $\mathbf{A}_{l_{n}} \mathbf{T}^{n-1}$ and $j \mathbf{A}_{l_{n}} \mathbf{T}^{n-1}$, respectively, and those 
associated with the symbols $s_{m_{I}}$ and $s_{m_{Q}}$ are $\mathbf{A}_{l_{m}} \mathbf{T}^{m-1}$ and $j \mathbf{A}_{l_{m}} \mathbf{T}^{m-1}$, respectively, it can be readily seen that if $\left(\mathbf{A}_{l_{n}} \mathbf{T}^{n-1}\right) \perp\left(\mathbf{A}_{l_{m}} \mathbf{T}^{m-1}\right)$, then $\left(j \mathbf{A}_{l_{n}} \mathbf{T}^{n-1}\right) \perp\left(\mathbf{A}_{l_{m}} \mathbf{T}^{m-1}\right)$ and $\left(j \mathbf{A}_{l_{n}} \mathbf{T}^{n-1}\right) \perp\left(j \mathbf{A}_{l_{m}} \mathbf{T}^{m-1}\right)$.

Lemma 1: For any $\mathbf{A}_{i}, \mathbf{A}_{j} \in \mathcal{B},\left(\mathbf{A}_{i} \mathbf{T}^{l}\right) \perp\left(\mathbf{A}_{j} \mathbf{T}^{l+k}\right)$ i.e. $\left(\mathbf{A}_{i} \mathbf{T}^{l}\right)\left(\mathbf{A}_{j} \mathbf{T}^{l+k}\right)^{H}+\left(\mathbf{A}_{j} \mathbf{T}^{l+k}\right)\left(\mathbf{A}_{i} \mathbf{T}^{l}\right)^{H}=\mathbf{O}$ for $P \leq k \leq$ $K-l-1$ and $0 \leq l \leq K-1$.

Proof: Proof is provided in Appendix B.

Lemma 2: In the ZP-SC SM system, the extended encoding groups $\mathbf{x}_{n}^{\prime}$ and $\mathbf{x}_{m}^{\prime}$ are non-interfering if $n+P \leq m$.

Proof: Proof directly follows from Lemma 1 and is provided for the sake of completeness. The vertices $\mathbf{x}_{n}^{\prime}$ and $\mathbf{x}_{m}^{\prime}$ are non-interfering if $\left(\mathbf{A}_{l_{n}} \mathbf{T}^{n-1}\right) \perp\left(\mathbf{A}_{l_{m}} \mathbf{T}^{m-1}\right)$. From Lemma 1 we have $\left(\mathbf{A}_{l_{n}} \mathbf{T}^{n-1}\right) \perp\left(\mathbf{A}_{l_{m}} \mathbf{T}^{m-1}\right)$, if $m-1 \geq$ $n-1+P \Rightarrow m \geq n+P$.

Proposition 2: In the ZP-SC SM scheme having $N_{t}$ transmit antennas, the order ${ }^{3}$ of $\mathrm{ML}$ decoding complexity is $\left(N_{t} M\right)^{P}$, where $M$ is the size of the signal set employed and $P$ is the number of multipath links between each of the transmit and receive antenna pairs.

Proof: We show here that there exists a core $G^{\prime}$ for the junction tree of the ZP-SC SM system such that $\max _{v \in V}\left|\mathcal{A}_{\mathcal{I}_{v}}\right|=\left(N_{t} M\right)^{P}$. Note that if the vertices $\mathbf{x}_{n}^{\prime}$ and $\mathbf{x}_{m}^{\prime}$ are non-interfering, then the vertex corresponding to $\left(\mathbf{x}_{n}^{\prime}, \mathbf{x}_{m}^{\prime}\right)$ can be removed from the junction tree, since $\alpha_{n, m}^{\prime}\left(\mathbf{x}_{n}^{\prime}, \mathbf{x}_{m}^{\prime}\right)=0$. From Lemma 2 , the vertices corresponding to $\left(\mathbf{x}_{n}^{\prime}, \mathbf{x}_{m}^{\prime}\right)$ for $m \geq n+P$ are non-interfering and hence they do not have to be included in the junction tree. We propose a junction tree for the ZP-SC SM system shown in Fig. 2. It can be readily verified that the core of the junction tree given in Fig. 2 satisfies the condition C.1 described in Section III-A. Furthermore, it may be seen from Fig. 2 that the $\max _{v \in V}\left|\mathcal{A}_{\mathcal{I}_{v}}^{\prime}\right|=\left|\mathcal{A}_{n}^{\prime}\right|^{P}=|S \times L|^{P}=\left(N_{t} M\right)^{P}$.

Although the order of ML decoding complexity is reduced from $\left(N_{t} M\right)^{K}$ to $\left(N_{t} M\right)^{P}$, the complexity still remains high for the number of multipaths encountered in practical dispersive scenarios. For example, the Vehicular A and Pedestrian B channel models specified for cellular communication by 3GPP consider $P=6$ links [39], while some HIPERLAN channel models even consider $P=16$ links [40]. For the modest values of $N_{t}=M=2$ and $P=6$, we will have $\left(N_{t} M\right)^{P}=4096$. Thus, the ML decoding in the ZP-SC SM system is only feasible when $P$ is small. When $P$ is large, we may have to resort to low-complexity sub-optimal linear receivers.

\section{A LOW-COMPLEXITY LINEAR RECEIVER FOR ZP-SC SYSTEMS THAT ACHIEVE FULL TRANSMIT, RECEIVE AND MULTIPATH DIVERSITY}

In the previous section, we showed that the order of ML decoding complexity in the ZP-SC SM system is $\left(N_{t} M\right)^{P}$. The length of the multipath channel $P$ may be reduced to a more modest value with the aid of channel shortening filters [41], [42]. However, channel shortening does not necessarily guarantee attaining full multipath diversity. In this section, we

\footnotetext{
${ }^{3}$ Given an optimization problem, the number of evaluations of the optimization metric is defined as the order of complexity for the algorithm.
}

propose a low-complexity PIC-R for the general ZP-SC communication system that converts the mulitpath system into a set of frequency-flat block fading subsystems. Furthermore, we show that the transmission of any full-rank STBC over these subsystems achieves full transmit-, receive- and multipathdiversity under ML decoding. Finally, we show that the SM scheme can be viewed as a pure spatial-domain arrangement, and hence achieves only receive- and multipath-diversity under PIC-R, while imposing a complexity order of $N_{t} M$.

\section{A. Proposed PIC-R for the general ZP-SC system}

Assuming that the multipath channel's complex-valued envelope is fixed over $T^{\prime}$ data frames, we can extend the ZP-SC system of (3) over $T^{\prime}$ data frames as

$$
\hat{\mathbf{Y}}=\hat{\mathbf{H}} \hat{\mathbf{X}}+\hat{\mathbf{N}},
$$

where $\hat{\mathbf{Y}}=\left[\hat{\mathbf{y}}_{1}, \hat{\mathbf{y}}_{2}, \ldots, \hat{\mathbf{y}}_{T^{\prime}}\right], \hat{\mathbf{X}}=\left[\hat{\mathbf{x}}_{1}, \hat{\mathbf{x}}_{2}, \ldots, \hat{\mathbf{x}}_{T^{\prime}}\right]$ and $\hat{\mathbf{N}}=$ $\left[\hat{\mathbf{n}}_{1}, \hat{\mathbf{n}}_{2}, \ldots, \hat{\mathbf{n}}_{T^{\prime}}\right]$. Let

$$
\mathcal{I}_{i}=\left\{N_{t} i+1, N_{t} i+2, \ldots, N_{t}(i+1)\right\},
$$

for $i=0,1, \cdots, K-1$, such that $\cup_{i=0}^{K-1} \mathcal{I}_{i}=\mathcal{I}=\{i\}_{i=1}^{N_{t} K}$ and $\mathbf{G}_{\mathcal{I}_{i}}$ be the matrix having columns of $\hat{\mathbf{H}}$ that are indexed by the elements of $\mathcal{I}_{i}$. Then (12) may be equivalently written as

$$
\hat{\mathbf{Y}}=\sum_{i=0}^{K-1} \mathbf{G}_{\mathcal{I}_{i}} \hat{\mathbf{X}}_{i}+\hat{\mathbf{N}}
$$

where $\hat{\mathbf{X}}_{i}=\hat{\mathbf{X}}\left(\left[i N_{t}+1:(i+1) N_{t}\right],:\right) \quad \in$ $\mathbb{C}^{N_{t} \times T^{\prime}}$, for $0 \leq i \leq K-1$. Let $\mathbf{G}_{\mathcal{I}_{k}}^{c}=$ $\left[\mathbf{G}_{\mathcal{I}_{0}}, \mathbf{G}_{\mathcal{I}_{1}}, \ldots, \mathbf{G}_{\mathcal{I}_{k-1}}, \mathbf{G}_{\mathcal{I}_{k+1}}, \ldots, \mathbf{G}_{\mathcal{I}_{K-1}}\right]$. The matrix projecting on to the orthogonal complement space of $\mathbf{G}_{\mathcal{I}_{k}}^{c}$ is given by $\mathbf{P}_{\mathcal{I}_{k}}=\mathbf{I}_{N_{r}(K+P-1)}-\mathbf{Q}_{\mathcal{I}_{k}}$, where $\mathbf{Q}_{\mathcal{I}_{k}}=$ $\mathbf{G}_{\mathcal{I}_{k}}^{c}\left(\left(\mathbf{G}_{\mathcal{I}_{k}}^{c}\right)^{H} \mathbf{G}_{\mathcal{I}_{k}}^{c}\right)^{-1}\left(\mathbf{G}_{\mathcal{I}_{k}}^{c}\right)^{H}$. Thus, we have $\mathbf{P}_{\mathcal{I}_{k}} \mathbf{G}_{\mathcal{I}_{i}}=\mathbf{0}$ for $i \in\{j\}_{j=0}^{K-1} \backslash k$. Consider $\mathbf{Z}_{\mathcal{I}_{k}}=\mathbf{P}_{\mathcal{I}_{k}} \hat{\mathbf{Y}}$ given by

$$
\begin{aligned}
\mathbf{Z}_{\mathcal{I}_{k}} & =\mathbf{P}_{\mathcal{I}_{k}} \sum_{i=1}^{K-1} \mathbf{G}_{\mathcal{I}_{i}} \hat{\mathbf{X}}_{i}+\mathbf{P}_{\mathcal{I}_{k}} \hat{\mathbf{N}}, \\
& =\mathbf{P}_{\mathcal{I}_{k}} \mathbf{G}_{\mathcal{I}_{k}} \hat{\mathbf{X}}_{k}+\mathbf{P}_{\mathcal{I}_{k}} \hat{\mathbf{N}} .
\end{aligned}
$$

The PIC-R solution for the $k^{\text {th }}$ subsystem is given by

$$
\left(\hat{\mathbf{X}}_{k}\right)_{P I C-R}=\arg \min _{\mathbf{X} \in \mathcal{C}^{\prime}}\left\|\mathbf{Z}_{\mathcal{I}_{k}}-\mathbf{P}_{\mathcal{I}_{k}} \mathbf{G}_{\mathcal{I}_{k}} \mathbf{X}\right\|^{2},
$$

for $0 \leq k \leq K-1$.

Proposition 3: The ZP-SC system having $N_{t}$ transmit and $N_{r}$ receive antennas associated with $P$ multipath links between each transmit and receive antenna pair achieves a diversity order of $N_{t} N_{r} P$ with the aid of PIC-R under the grouping scheme $\mathcal{I}_{i}$ given in (13), when each $\hat{\mathbf{X}}_{k}$ is from a full-rank space-time codebook $\mathcal{C}^{\prime}$.

Proof: Proof is provided in Appendix C.

Example 2: Considering the system presented in Example 1 , we have $\mathcal{I}_{0}=\{1,2\}, \mathcal{I}_{1}=\{3,4\}, \mathcal{I}_{2}=\{5,6\}$, and hence

$$
\mathbf{G}_{\mathcal{I}_{0}}=\left[\begin{array}{cc}
\mathbf{h}_{0}(1) & \mathbf{h}_{0}(2) \\
\mathbf{h}_{1}(1) & \mathbf{h}_{1}(2) \\
\mathbf{h}_{2}(1) & \mathbf{h}_{2}(2) \\
0 & 0 \\
0 & 0
\end{array}\right], \mathbf{G}_{\mathcal{I}_{1}}=\left[\begin{array}{cc}
0 & 0 \\
\mathbf{h}_{0}(1) & \mathbf{h}_{0}(2) \\
\mathbf{h}_{1}(1) & \mathbf{h}_{1}(2) \\
\mathbf{h}_{2}(1) & \mathbf{h}_{2}(2) \\
0 & 0
\end{array}\right]
$$




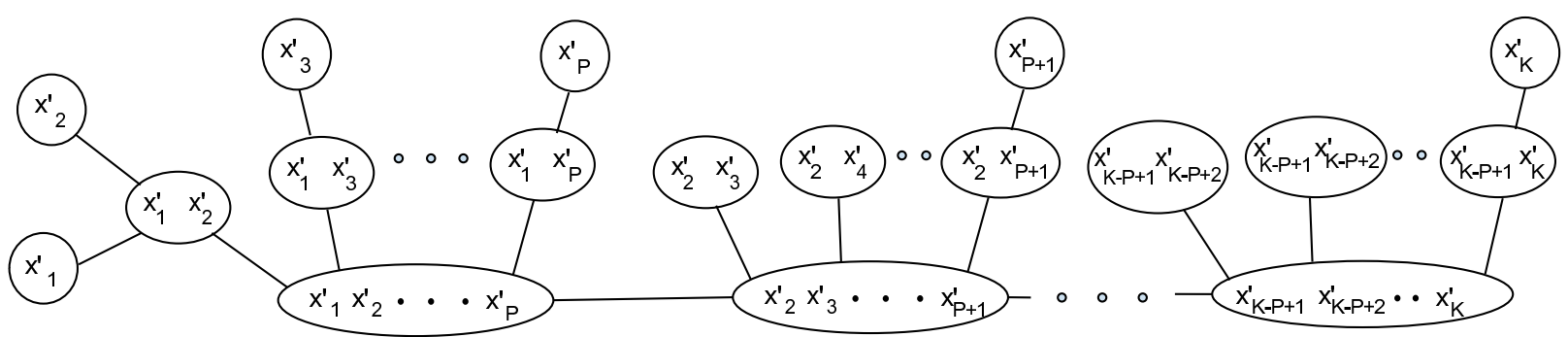

Fig. 2. Proposed junction tree for ML decoding in ZP-SC SM system.

and

$$
\mathbf{G}_{\mathcal{I}_{2}}=\left[\begin{array}{cc}
0 & 0 \\
0 & 0 \\
\mathbf{h}_{0}(1) & \mathbf{h}_{0}(2) \\
\mathbf{h}_{1}(1) & \mathbf{h}_{1}(2) \\
\mathbf{h}_{2}(1) & \mathbf{h}_{2}(2)
\end{array}\right] .
$$

With this grouping scheme, we have $\mathbf{G}_{\mathcal{I}_{0}}^{c}=\left\{\mathbf{G}_{\mathcal{I}_{1}}, \mathbf{G}_{\mathcal{I}_{2}}\right\}$, $\mathbf{G}_{\mathcal{I}_{1}}^{c}=\left\{\mathbf{G}_{\mathcal{I}_{0}}, \mathbf{G}_{\mathcal{I}_{2}}\right\}$ and $\mathbf{G}_{\mathcal{I}_{2}}^{c}=\left\{\mathbf{G}_{\mathcal{I}_{0}}, \mathbf{G}_{\mathcal{I}_{1}}\right\}$. Furthermore, $\mathbf{Z}_{\mathcal{I}_{k}}$ for $k=0,1,2$ are computed as per (16) and the STBCs transmitted over each subsystem are detected according to (17).

Corollary 1: With the grouping scheme $\mathcal{I}_{i}$ given in (13), the ZP-SC SM system achieves a diversity order of $N_{r} P$ and a decoding complexity order of $N_{t} M$ with the aid of the PIC$\mathrm{R}$.

Proof: It is clear from (2) that the vector transmitted in the SM system is from the set of length $N_{t}$ vectors given by

$$
\mathcal{C}_{S M}^{\prime}=\left\{\left[\begin{array}{c}
s \\
0 \\
0 \\
\vdots \\
0
\end{array}\right],\left[\begin{array}{c}
0 \\
s \\
0 \\
\vdots \\
0
\end{array}\right],\left[\begin{array}{c}
0 \\
0 \\
s \\
\vdots \\
0
\end{array}\right], \cdots \cdots,\left[\begin{array}{c}
0 \\
0 \\
0 \\
\vdots \\
s
\end{array}\right]\right\},
$$

where $s \in S$ is from an $M$-QAM or -PSK constellation. Furthermore, it may be readily seen that any element in $\Delta \mathcal{C}_{S M}^{\prime}=\left\{\mathbf{X}_{1}-\mathbf{X}_{2} \mid \mathbf{X}_{1} \neq \mathbf{X}_{2}, \mathbf{X}_{1}, \mathbf{X}_{2} \in \mathcal{C}_{S M}^{\prime}\right\}$ is of rankone. Thus, $\mathcal{C}_{S M}^{\prime}$ may be viewed as a rank-one STBC, and hence it follows from Proposition 3 that the ZP-SC SM system achieves a diversity order of $N_{r} P$. Since we have $\left|\mathcal{C}_{S M}^{\prime}\right|=N_{t} M$, from (17) it is straightforward to show that the order of decoding complexity is $N_{t} M$. This concludes the proof.

\section{B. PIC-R with Successive Interference Cancellation (PIC-R- SIC) for the general ZP-SC system}

The PIC-R algorithm conceived for the ZP-SC system presented in the previous subsection can be further improved by canceling the interference from the already detected STBCstreams in a successive fashion leading to an algorithm similar to the one presented in [27]. However, limited attention was dedicated in [27] to the order in which the information of the various groups is detected. We show that the order in which the interference is canceled in the ZP-SC system can be adjusted for significantly reducing the computational complexity of the PIC-R-SIC. Specifically, we exploit the displacement structure [31]-[33] of the the block Toeplitz $\hat{\mathbf{H}}$ and reduce the complexity involved in computing each $\mathbf{P}_{\mathcal{I}_{k}}$. Furthermore, we show in the next section that the PIC-R-SIC is capable of attaining significant symbol error ratio (SER) gains with respect to the PIC-R operating without SIC.

The proposed ordering of the groups for the PIC-R-SIC in the $\mathrm{ZP}-\mathrm{SC}$ system is as follows:

$$
\left\{\mathcal{I}_{K-1}, \mathcal{I}_{K-2}, \ldots, \mathcal{I}_{1}, \mathcal{I}_{0}\right\} .
$$

Algorithm 1 outlines the PIC-R-SIC, which cancels the interference from the STBCs in the order given in (19). Computational burden imposed by Algorithm 1 is mainly dominated by (20) and (21). The computational complexity due to (21) depends on the specific choice of the STBC $\mathcal{C}^{\prime}$, while that due to (20) depends only on $\hat{\mathbf{H}}$. First, we focus our attention on reducing the complexity involved in computing (20).

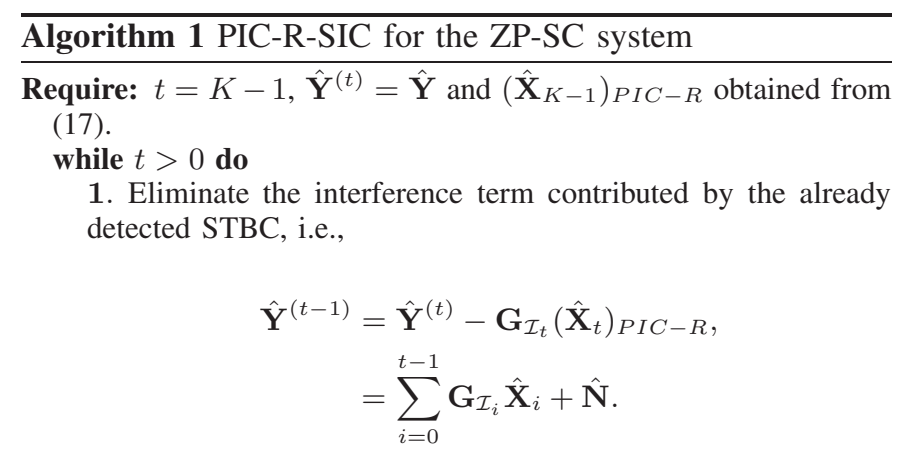

2. Obtain the projection matrix $\mathbf{P}_{\mathcal{I}_{t-1}}=\mathbf{I}-\mathbf{Q}_{\mathcal{I}_{t-1}}$, where

$$
\mathbf{Q}_{\mathcal{I}_{t-1}}=\mathbf{G}_{\mathcal{I}_{t-1}}^{c}\left(\left(\mathbf{G}_{\mathcal{I}_{t-1}}^{c}\right)^{H} \mathbf{G}_{\mathcal{I}_{t-1}}^{c}\right)^{-1}\left(\mathbf{G}_{\mathcal{I}_{t-1}}^{c}\right)^{H}
$$

such that

$$
\mathbf{G}_{\mathcal{I}_{t-1}}^{c}=\left[\mathbf{G}_{\mathcal{I}_{0}}, \mathbf{G}_{\mathcal{I}_{1}}, \ldots, \mathbf{G}_{\mathcal{I}_{t-2}}\right]
$$

3. Obtain

$$
\begin{aligned}
\mathbf{Z}_{\mathcal{I}_{t-1}} & =\mathbf{P}_{\mathcal{I}_{t-1}} \sum_{i=0}^{t-1} \mathbf{G}_{\mathcal{I}_{i}} \hat{\mathbf{X}}_{i}+\mathbf{P}_{\mathcal{I}_{t-1}} \hat{\mathbf{N}} \\
& =\mathbf{P}_{\mathcal{I}_{t-1}} \mathbf{G}_{\mathcal{I}_{t-1}} \hat{\mathbf{X}}_{t-1}+\mathbf{P}_{\mathcal{I}_{t-1}} \hat{\mathbf{N}}
\end{aligned}
$$

and

$$
\left(\hat{\mathbf{X}}_{t-1}\right)_{P I C-R}=\arg \min _{\mathbf{X} \in \mathcal{C}^{\prime}}\left\|\mathbf{Z}_{\mathcal{I}_{t-1}}-\mathbf{P}_{\mathcal{I}_{t-1}} \mathbf{G}_{\mathcal{I}_{t-1}} \mathbf{X}\right\|^{2} .
$$

4. $t \rightarrow t-1$.

end while

In the $l^{\text {th }}$ iteration of Algorithm 1, we have to com- 
pute $\mathbf{Q}_{\mathcal{I}_{l}}=\mathbf{G}_{\mathcal{I}_{l}}^{c}\left(\left(\mathbf{G}_{\mathcal{I}_{l}}^{c}\right)^{H} \mathbf{G}_{\mathcal{I}_{l}}^{c}\right)^{-1}\left(\mathbf{G}_{\mathcal{I}_{l}}^{c}\right)^{H}$, where $\mathbf{G}_{\mathcal{I}_{l}}^{c}=$ $\left[\mathbf{G}_{\mathcal{I}_{0}}, \mathbf{G}_{\mathcal{I}_{1}}, \ldots, \mathbf{G}_{\mathcal{I}_{l-1}}\right] \in \mathbb{C}^{N_{r}(K+P-1) \times N_{t} l}$. For simplifying our complexity analysis, we assume $N_{r}=N_{t}$, where the complexity is quantified in terms of the number of multiplications/additions. Direct computation of $\mathbf{Q}_{\mathcal{I}_{l}}$ without exploiting the structure or sparsity of $\hat{\mathbf{H}}$ results in a computational complexity of order $\mathcal{O}\left(N_{t}^{3} l^{3}\right)$. Since the maximum value of $l$ is $K-1$, the complexity order involved in computing the set $\left\{\mathbf{Q}_{\mathcal{I}_{k}}\right\}_{k=0}^{K-1}$ is $\mathcal{O}\left(N_{t}^{3} K^{3}\right)$. In what follows, we give a brief outline of the theory of displacement structures and show that the order of complexity involved in computing the set $\left\{\mathbf{Q}_{\mathcal{I}_{k}}\right\}_{k=0}^{K-1}$ can be reduced to $\mathcal{O}\left(N_{t}^{3} K \log ^{2} K\right)$.

Consider $\mathbf{C} \in \mathbb{C}^{m \times n}$ and strictly lower triangular matrices $\mathbf{F}^{f} \in \mathbb{R}^{m \times m}$ and $\mathbf{F}^{b} \in \mathbb{R}^{n \times n}$.

1) The displacement of $\mathbf{C}$ with respect to the displacement operators $\mathbf{F}^{f}$ and $\mathbf{F}^{b}$ is given by $\nabla_{\left(\mathbf{F}^{f}, \mathbf{F}^{b}\right)} \mathbf{C}=\mathbf{C}-$ $\mathbf{F}^{f} \mathbf{C}\left(\mathbf{F}^{b}\right)^{T}$.

2) The matrix pair $\mathbf{J}=\left[\mathbf{j}_{1}, \mathbf{j}_{2}, \ldots, \mathbf{j}_{\alpha}\right]$ and $\mathbf{K}=$ $\left[\mathbf{k}_{1}, \mathbf{k}_{2}, \cdots, \mathbf{k}_{\alpha}\right]$ is said to be a generator of $\mathbf{C}$ if we have $\nabla_{\left(\mathbf{F}^{f}, \mathbf{F}^{b}\right)} \mathbf{C}=\mathbf{J K}^{H}$, and $\alpha$ is referred to as the length of the generator. The pair $(\mathbf{J}, \mathbf{K})$ with minimal possible length is termed as the minimal generator and its length is referred to as the displacement rank of $\mathbf{C}$ denoted by $\alpha_{d i s}$.

3) The displacement representation of $\mathbf{C}$ is given by

$$
\mathbf{C}=\sum_{i=1}^{\alpha} \mathbf{L}_{n}\left(\mathbf{j}_{i}, \mathbf{F}^{f}\right) \mathbf{L}_{n}^{H}\left(\mathbf{k}_{i}, \mathbf{F}^{b}\right),
$$

where $\mathbf{L}_{n}\left(\mathbf{j}_{i}, \mathbf{F}^{f}\right) \in \mathbb{C}^{m \times n}$ and $\mathbf{L}_{n}\left(\mathbf{k}_{i}, \mathbf{F}^{b}\right) \in$ $\mathbb{C}^{n \times n}$ are the lower triangular matrices given by $\mathbf{L}_{n}\left(\mathbf{j}_{i}, \mathbf{F}^{f}\right)=\left[\mathbf{j}_{i}, \mathbf{F}^{f} \mathbf{j}_{i}, \ldots,\left(\mathbf{F}^{f}\right)^{n-1} \mathbf{j}_{i}\right], \mathbf{L}_{n}\left(\mathbf{k}_{i}, \mathbf{F}^{b}\right)=$ $\left[\mathbf{k}_{i}, \mathbf{F}^{b} \mathbf{k}_{i}, \ldots,\left(\mathbf{F}^{b}\right)^{n-1} \mathbf{k}_{i}\right]$.

Lemma 3 (Chun and Kailath): If $\mathbf{A}=\left[\begin{array}{ll}\mathbf{A}_{1,1} & \mathbf{A}_{1,2} \\ \mathbf{A}_{2,1} & \mathbf{A}_{2,2}\end{array}\right] \in$ $\mathbb{C}^{m \times n}$, has a displacement rank $\alpha_{d i s}$ with respect to the displacement operators $\mathbf{F}^{f}$ and $\mathbf{F}^{b}$, then the generator of the Schur complement of $\mathbf{A}_{1,1} \in \mathbb{C}^{r \times r}$, i.e. $\mathbf{A}_{2,2}-\mathbf{A}_{2,1} \mathbf{A}_{1,1}^{-1} \mathbf{A}_{1,2}$, can be obtained by $r$ recursions of the Generalized Schur Algorithm (refer to Section 2 of [31]) with a computational complexity order of $\mathcal{O}\left(\alpha_{d i s}^{3} r \log ^{2} r\right)$.

Proof: Proof can be found in Section 4 of [31].

Let us now consider $\mathbf{Q}_{\mathcal{I}_{l}}=\mathbf{G}_{\mathcal{I}_{l}}^{c}\left(\left(\mathbf{G}_{\mathcal{I}_{l}}^{c}\right)^{H} \mathbf{G}_{\mathcal{I}_{l}}^{c}\right)^{-1}\left(\mathbf{G}_{\mathcal{I}_{l}}^{c}\right)^{H}$, which represents the Schur complement of the $(1,1)$ block of $\overline{\mathbf{Q}}_{\mathcal{I}_{l}}=\left[\begin{array}{cc}\left(\mathbf{G}_{\mathcal{I}_{l}}^{c}\right)^{H} \mathbf{G}_{\mathcal{I}_{l}}^{c} & \left(\mathbf{G}_{\mathcal{I}_{l}}^{c}\right)^{H} \\ -\mathbf{G}_{\mathcal{I}_{l}}^{c} & \mathbf{O}\end{array}\right] \in$ $\mathbb{C}^{\left(N_{t} K+N_{r}\{K+P-1\}\right) \times\left(N_{t} K+N_{r}\{K+P-1\}\right)}$. By choosing $\mathbf{F}^{f}=\mathbf{F}^{b}=\left[\begin{array}{cc}\mathbf{W}_{1} & \mathbf{O} \\ \mathbf{O} & \mathbf{W}_{2}\end{array}\right]$, where $\mathbf{W}_{1} \in \mathbb{R}^{N_{t} l \times N_{t} l}$ is a matrix with $\mathbf{I}_{N_{t}}$ on the subdiagonal blocks and $\mathbf{O}$ elsewhere, while $\mathbf{W}_{2} \in \mathbb{R}^{N_{r}(K+P-1) \times N_{r}(K+P-1)}$ is a matrix with $\mathbf{I}_{N_{r}}$ on the subdiagonal blocks and $\mathbf{O}$ elsewhere, one can verify that $\alpha_{d i s}$ of $\overline{\mathbf{Q}}_{\mathcal{I}_{l}}$ is $2 N_{t}$. Thus, from Lemma 3 we can obtain $\mathbf{Q}_{\mathcal{I}_{l}}$ in $l$ recursions of the generalized Schur algorithm associated with a complexity order $\mathcal{O}\left(N_{t}^{3} l \log ^{2} l\right)$. Since, the maximum value that $l$ can assume is $K-1$, the complexity order is approximately equal to $\mathcal{O}\left(N_{t}^{3} K \log ^{2} K\right)$. Note that the ordering given in (19) enables us to reuse the principal sub-matrix blocks of $\overline{\mathbf{Q}}_{\mathcal{I}_{K-1}}$ for the computation of $\overline{\mathbf{Q}}_{\mathcal{I}_{l}}$ for

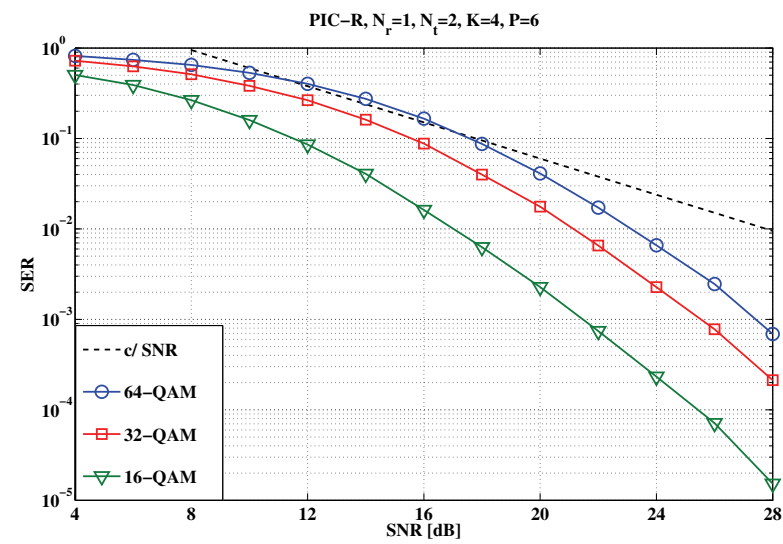

Fig. 3. SER performance of the ZP-SC SM system with PIC-R having $N_{t}=2, N_{r}=1, K=4, P=6$, and employing 16-, 32- and 64-QAM signal sets.

$0 \leq l \leq K-2$, and hence facilitates further complexity reduction.

Remark 2: Note that by exploiting the block Toeplitz structure of $\hat{\mathbf{H}}$, the order of computational complexity of the ZF/MMSE equalizer based ZP-SC receivers can also be reduced to $\mathcal{O}\left(N_{t}^{3} K \log ^{2} K\right)$ (refer to chapter 6 of [46]). Thus, the order of computational complexity in the proposed PIC$\mathrm{R}$-SIC is the same as that of the ZF/MMSE equalizer based ZP-SC receivers.

It may be readily seen that the order of complexity involved in computing (21) is $\left|\mathcal{C}^{\prime}\right|$. When employing $\mathrm{SM}$, we have $\mathcal{C}^{\prime}$ equal to $\mathcal{C}_{S M}^{\prime}$, which is given by (18). Thus, the order of computational complexity of (21) is $\left|\mathcal{C}_{S M}^{\prime}\right|=N_{t} M$. When the signal set employed is a square or a rectangular QAM, the order of decoding complexity can be reduced to $N_{t}$ with the aid of hard-limiting. For further details, please refer to Proposition 1 of [8].

\section{Simulation RESUlts}

Simulation scenario: In all our simulations, we considered an SER of $10^{-t}$ and used at least $10^{t+2}$ symbols in evaluating the SER. Furthermore, we assumed block Rayleigh fading multipath channels with a uniform power delay profile. All the receivers considered in our simulations are assumed to have perfect channel state information.

First, we validate our claim that the proposed PIC-R aided ZP-SC SM system is capable of achieving multipath diversity. Consider a ZP-SC SM system having $N_{t}=2, N_{r}=1, K=4$, $P=6$ and employing the PIC-R. Fig. 3 portrays the SER performance of this system, when considering 16-, 32- and 64QAM. In Fig. 3, the curve corresponding to the function $\frac{c}{S N R}$ serves as a reference corresponding to a diversity order one. It is clear from Fig. 3 that the PIC-R is capable of achieving multipath diversity.

In order to show the superiority of PIC-R-SIC over PIC-R, we consider a ZP-SC SM system having $N_{t}=2, N_{r}=2$, $K=8$ and employing 16- and 64-QAM signal sets. Fig. 4 compares the SER performance of both PIC-R-SIC and PICR. It is clear from Fig. 4 that the PIC-R-SIC gives a better SER performance than the PIC-R associated with both the signal 


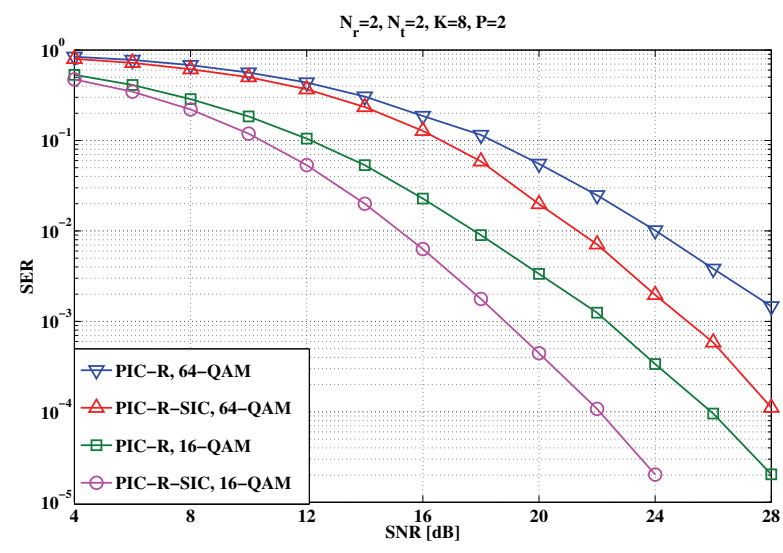

Fig. 4. SER performance of the ZP-SC SM system with PIC-R-SIC and PIC-R having $N_{t}=2, N_{r}=2, K=8, P=2$, and employing 16- and 64-QAM signal sets.

sets. Specifically, an SNR gain of about $3.5 \mathrm{~dB}$ is observed at an SER of about $10^{-4}$ for the 64-QAM signal set, and about $4 \mathrm{~dB}$ at an SER of about $10^{-3}$ for the 16-QAM signal set is observed.

We consider the ZP-SC, the CP-SC and the cyclic prefixed Orthogonal Frequency Division Multiplexing (CP-OFDM) systems, all employing SM and having identical $N_{t}, N_{r}, K$ and throughput values. We compare their SER performances. The ZP-SC system is considered with the proposed PIC-RSIC, the conventional ZF and the MMSE equalizer based receivers. The CP-SC system is considered in conjunction with the ZF and MMSE based receivers. The SM symbols generated after equalization (in both the ZF and MMSE modes) are detected by minimizing the Euclidean distance between the received and the legitimate transmit vectors. In CP-OFDM system, the SM symbols are detected in the frequency domain based on the minimum Euclidean distance metric.

Consider the ZP-SC SM, CP-SC SM, and the CP-OFDM SM systems having $N_{t}=4, N_{r}=4, K=8$ and employing 16- and 64-QAM signal sets. Fig. 5 and Fig. 6 compare the SER performance of these systems along with the various receivers considered above. It is clear from both Fig. 5 and Fig. 6 that the SER performance of SM in the ZP-SC system is significantly better than that in the CP-OFDM or CPSC systems. It is evident from Fig. 5 and Fig. 6 that the CP-SC system performs poorer than the CP-OFDM system. Furthermore, it is clear from Fig. 5 and Fig. 6 that the proposed PIC-R-SIC achieves significant performance gains over the conventional $\mathrm{ZF}$ and MMSE equalizer based receivers. Specifically, when using the 16-QAM signal set, the proposed PIC-R-SIC achieves an SNR gain of about $6 \mathrm{~dB}$ over the ZF and MMSE equalizer based receivers at an SER of about $10^{-4}$ and about $11 \mathrm{~dB}$ over the CP-OFDM SM system. When using the 64-QAM signal set, the proposed PIC-R-SIC achieves an SNR gain of about $6 \mathrm{~dB}$ over the ZF and MMSE equalizer based receivers at an SER of about $10^{-3}$ and about $10 \mathrm{~dB}$ over the CP-OFDM SM system. It can be observed from Fig. 5 and Fig. 6 that the SM system employing ZF/MMSE based receivers is capable of achieving a considerable diversity order.

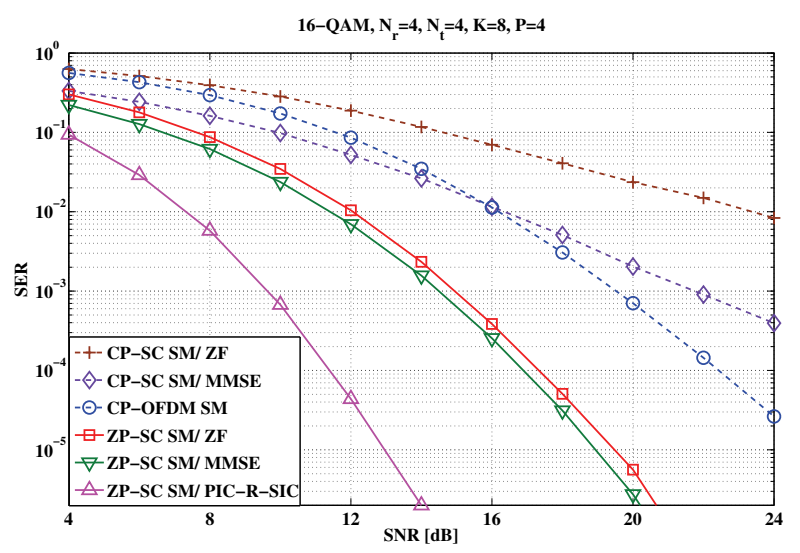

Fig. 5. SER performance of the CP-OFDM SM system, the CP-SC SM system employing ZF and MMSE equalizer based receivers, and of the ZPSC SM systems employing the PIC-R-SIC, ZF, and MMSE based receivers. All the systems are assumed to have $N_{t}=4, N_{r}=4, K=8, P=4$ and operating with 16-QAM signal set.

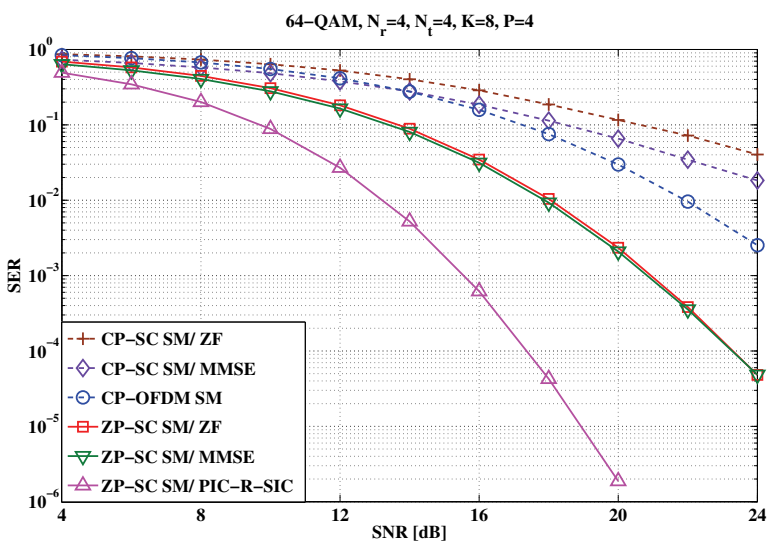

Fig. 6. SER performance of the CP-OFDM SM system, the CP-SC SM system employing $\mathrm{ZF}$ and MMSE equalizer based receivers, and of the $\mathrm{ZP}$ SC SM systems employing the PIC-R-SIC, ZF, and MMSE based receivers. All the systems are assumed to have $N_{t}=4, N_{r}=4, K=8, P=4$ and operating with 64-QAM signal set.

An intuitive explanation for this is that the set containing the differences of all possible transmit vectors in the SM system forms a subset of its C-MIMO counter part and C-MIMO is known to achieve a diversity order of $N_{r} P-N_{t}+1$ with the aid of the ZF/MMSE based receiver [37].

In Fig. 7 and Fig. 8 we compare the SER performance of the ZP-SC SM system to those of the ZP-SC SIMO system $\left(N_{t}=1, N_{r}=4\right)$ and the ZP-SC C-MIMO system $\left(N_{t}=2,4\right.$ and $\left.N_{r}=4\right)$ operating at 6 bpcu and 8 bpcu, respectively. It can be readily seen from Fig. 7 and Fig. 8 that the SM system outperforms the SIMO system at all SNRs. Specifically, at a SER of $10^{-3}$ the SM system achieves an SNR gain of about 4 $\mathrm{dB}$ compared to the SIMO system operating at both the rates of 6 bpcu and 8 bpcu. It becomes clear from Fig. 7 that the SER performance of the SM system is nearly the same as those of the C-MIMO systems having $N_{t}=2$ and $N_{t}=3$, and it is slightly better than that of the $2 \times 4 \mathrm{C}$-MIMO system. On the other hand, it is evident from Fig. 8 that both the CMIMO systems outperform the SM system. Specifically, at an 


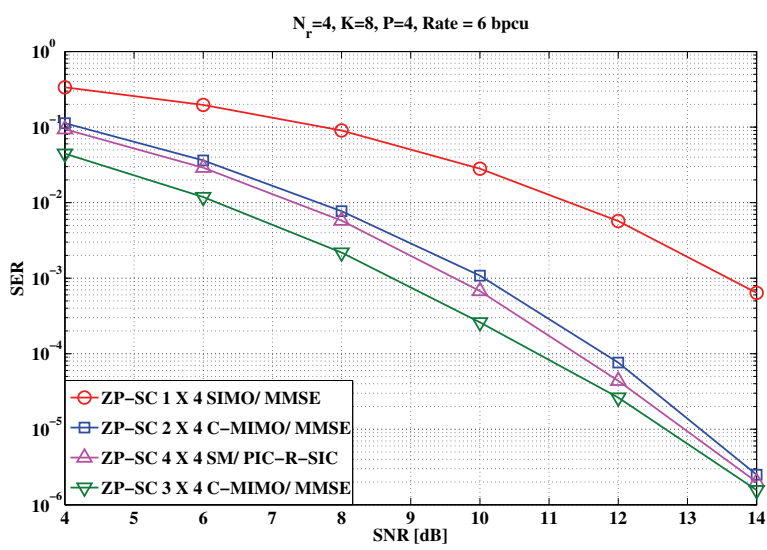

Fig. 7. SER performance of the ZP-SC $1 \times N_{r}$ SIMO system, the ZP-SC $N_{t} \times N_{r}$ C-MIMO system for various $N_{t}$ and the ZP-SC $N_{t} \times N_{r}$ SM system. All the systems are assumed to have $N_{r}=4, K=8, P=4$ and operating at a rate of 6 bpcu.

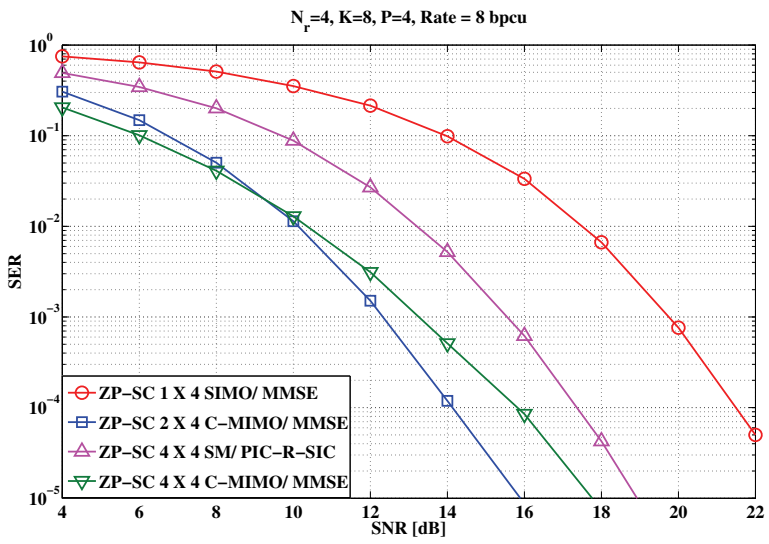

Fig. 8. SER performance of the ZP-SC $1 \times N_{r}$ SIMO system, the ZP-SC $N_{t} \times N_{r}$ C-MIMO system for various $N_{t}$ and the ZP-SC $N_{t} \times N_{r} \mathrm{SM}$ system. All the systems are assumed to have $N_{r}=4, K=8, P=4$ and operating at a rate of $8 \mathrm{bpcu}$.

SNR of $10^{-4}$ the $4 \times 4$ and $2 \times 4$ C-MIMO systems achieve an SNR gain of about $3 \mathrm{~dB}$ and $1 \mathrm{~dB}$ with respect to the SM system. Thus, we infer from Fig. 7 and Fig. 8 that the SM system is suitable for low and moderate throughputs and can be expected to give a comparable performance to that of the $C$-MIMO systems, despite having only one RF chain at the transmitter.

In order to achieve a high bandwidth efficiency, it is imperative to have large frame lengths. We increase the frame length $K$ and study the SER-variation of the proposed PIC-RSIC in the ZP-SC SM system. Fig. 9 portrays the change in the SER performance of the ZP-SC SM system having $N_{r}=4$, $N_{t}=4, P=4$, when $K$ takes the values 8,16, 32 and 64 . The degradation in SER performance as $K$ is increased from 8 to 64 is observed to be about $1 \mathrm{~dB}$ at an SER of $10^{-3}$.

\section{CONCLUSiOnS}

We have considered zero-padding instead of using a cyclic prefix for our SM aided single carrier system communicating over a frequency selective channel and shown that unlike the

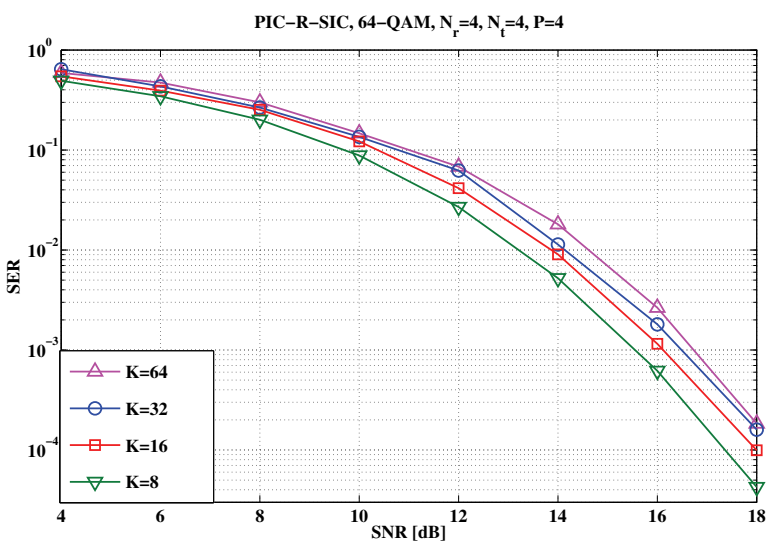

Fig. 9. SER performance curves of the ZP-SC SM system under PIC-RSIC for various frame lengths. The ZP-SC SM system considered here has $N_{t}=4, N_{r}=4, P=4$ and employs 64-QAM signal set.

cyclic prefixed SM system the zero-padded SM scheme offers full multipath diversity . Further, the order of ML decoding complexity in the zero-padded SM system is shown to be independent of the frame length and it is given by $\left(N_{t} M\right)^{P}$, where $P$ is the number of multipath links. This is in contrast to the cyclic prefixed SM system, whose ML decoding complexity order is exponential in the frame length, i.e. $\left(N_{t} M\right)^{K}$, where $K$ is the length of the data-frame. A low-complexity partial interference cancellation based linear receiver was proposed for the ZP-SC system, which achieves full multipath, transmit- and receive-diversity, when the transmitted STBC is of full-rank. Furthermore, it was shown that the SM aided ZP$\mathrm{SC}$ system is capable of achieving both multipath and receivediversity for the proposed linear receiver, and gives significant SNR gains with respect to the CP-OFDM SM, CP-SC SM and ZP-SC SM systems employing ZF/MMSE equalizer based receivers.

\section{APPEndiX A PROOF OF PROPOSITION 1}

Considering the system given in (10), the pairwise error probability of the ML detector can be bounded as [43]

$$
\begin{aligned}
\operatorname{PEP}\left(\mathbf{X}_{1} \rightarrow \mathbf{X}_{2}\right) & \leq \frac{1}{\operatorname{det}\left(\mathbf{I}+\frac{\rho}{4} \Delta \mathbf{X} \Delta \mathbf{X}^{H}\right)}, \\
& =\frac{1}{\prod_{i=1}^{r}\left(1+\frac{\rho}{4} \lambda_{i}\right)},
\end{aligned}
$$

where $\Delta \mathbf{X}=\mathbf{X}_{1}-\mathbf{X}_{2} \neq \mathbf{O}, \mathbf{X}_{1}, \mathbf{X}_{2} \in \mathcal{C}$ and $\lambda_{i}$ for $i=1,2, \ldots, r$ are the eigenvalues of $\Delta \mathbf{X} \Delta \mathbf{X}^{H}$. It is straightforward from (23) that the SM system achieves a diversity order of $r$ if at least $r$ eigenvalues of $\Delta \mathbf{X} \Delta \mathbf{X}^{H}$ are positive for any distinct $\mathbf{X}_{1}, \mathbf{X}_{2} \in \mathcal{C}$. Thus, in order to prove that the ZP-SC SM scheme is capable of fully exploiting the attainable multipath diversity, it is sufficient to show that the rank of the difference of any two distinct matrices from $\mathcal{C}$ is at least $P$. Let $\mathbf{X}=\sum_{k=1}^{K} s_{k} \mathbf{B}_{k} \mathbf{T}^{k-1}, \mathbf{X}^{\prime}=\sum_{k=1}^{K} s_{k}^{\prime} \mathbf{B}_{k}^{\prime} \mathbf{T}^{k-1}$ and $\mathbf{X} \neq \mathbf{X}^{\prime}$. The difference of the matrices $\mathbf{X}$ and $\mathbf{X}^{\prime}$ is given by $\sum_{k=1}^{K} \Delta_{k} \mathbf{T}^{k-1} \neq \mathbf{O}$, where $\Delta_{k}=s_{k} \mathbf{B}_{k}-s_{k}^{\prime} \mathbf{B}_{k}^{\prime}$. Let us introduce furthermore $\mathcal{M}_{k}=\left\{\mathbf{A}_{i}, \mathbf{A}_{j} \mid \mathbf{B}_{k}=\mathbf{A}_{i}, \mathbf{B}_{k}^{\prime}=\mathbf{A}_{j}\right\}$. It may be readily shown from (7) that we have $\operatorname{rank}\left(\Delta_{k}\right)=P$ 
if $\Delta_{k} \neq \mathrm{O}$ for $1 \leq k \leq K$. Furthermore, we have $\operatorname{rank}\left(\sum_{k=1}^{l} \Delta_{k} \mathbf{T}^{k-1}\right) \geq P$ for $1 \leq l \leq K-1$, since $\Delta_{l} \mathbf{T}^{l}$ adds a linearly independent column to $\sum_{k=1}^{l-1} \Delta_{k} \mathbf{T}^{k-1}$ if $\mathcal{M}_{l} \cap\left\{\cup_{i=1}^{l-1} \mathcal{M}_{i}\right\} \neq \mathcal{M}_{l}$ and equality is achieved only when all the $\mathcal{M}_{i}$ 's are identical. Thus, either we have $\operatorname{rank}\left(\sum_{k=1}^{K} \Delta_{k} \mathbf{T}^{k-1}\right) \geq P$ or $\operatorname{rank}\left(\sum_{k=1}^{K} \Delta_{k} \mathbf{T}^{k-1}\right)=0$. For the rank to drop to zero, all the $\Delta_{k}$ 's have to be zero. Since $\mathbf{X} \neq \mathbf{X}^{\prime}$, there exists at least one $\Delta_{k} \neq \mathbf{O}$ and hence, we have $\operatorname{rank}\left(\sum_{k=1}^{K} \Delta_{k} \mathbf{T}^{k-1}\right) \geq P$. This concludes the proof.

\section{APPENDIX B PROOF OF LEMMA 1}

Let $\mathbf{F}=\mathbf{A}_{i} \mathbf{T}^{l}$ and $\mathbf{G}=\mathbf{A}_{j} \mathbf{T}^{l+k}$. We have to show that $\mathbf{F} \mathbf{G}^{H}+\mathbf{G F}^{H}=\mathbf{O}$, which is equivalent to showing that $\mathbf{F G}^{T}+\mathbf{G F}^{T}=\mathbf{O}$, since both $\mathbf{F}$ and $\mathbf{G}$ are real matrices. We have $\mathbf{F G}^{T}=\sum_{p=1}^{K+P-1} \mathbf{f}_{p} \mathbf{g}_{p}^{T}$, where $\left\{\mathbf{f}_{p}\right\}_{p=1}^{K+P-1}$ and $\left\{\mathbf{g}_{p}\right\}_{p=1}^{K+P-1}$ are the columns of $\mathbf{F}$ and $\mathbf{G}$, respectively. From (7) and (8), we have

$\mathbf{A}_{i} \mathbf{T}^{l}=$

$$
\left[\mathbf{O}_{N_{t} P \times l}, \mathbf{e}_{P(i-1)+1}, \ldots, \mathbf{e}_{P(i-1)+P}, \mathbf{O}_{N_{t} P \times(K-l-1)}\right],
$$

and

$\mathbf{A}_{j} \mathbf{T}^{l+k}=$

$\left[\mathbf{O}_{N_{t} P \times(l+k)}, \mathbf{e}_{P(j-1)+1}, \ldots, \mathbf{e}_{P(j-1)+P}, \mathbf{O}_{N_{t} P \times(K-l-k-1)}\right]$

It may be seen from (24) that $\mathbf{f}_{p}=\mathbf{0}$ for $1 \leq p \leq l$ and $l+P+1 \leq p \leq K+P-1, \mathbf{f}_{p} \neq \mathbf{0}$ for $l+1 \leq p \leq l+P$. From (25), it can be observed that $\mathbf{g}_{p}=\mathbf{0}$ for $1 \leq p \leq l+k$ and $l+k+P+1 \leq p \leq K+P-1, \mathbf{g}_{p} \neq \mathbf{0}$ for $l+k+1 \leq p \leq$ $l+k+P$. Thus, we have $\sum_{p=1}^{l+k} \mathbf{f}_{p} \mathbf{g}_{p}^{T}=\mathbf{O}$ if $k \geq P$, and hence $\mathbf{F G}^{T}=\sum_{p=1}^{K+P-1} \mathbf{f}_{p} \mathbf{g}_{p}^{T}=\mathbf{O}$. Since $\mathbf{G F}^{T}=\left(\mathbf{F G}^{T}\right)^{T}=\mathbf{O}$, we have $\mathbf{F} \mathbf{G}^{T}+\mathbf{G F}^{T}=\mathbf{O}$. Note that this is true for any $0 \leq l \leq K-1$ and $P \leq k \leq K-l-1$. This concludes the proof.

\section{Appendix C \\ PROOF OF PROPOSITION 3}

The proof proceeds along the same lines as the proof given in [27], [28]. Since the elements of $\mathbf{P}_{\mathcal{I}_{k}} \hat{\mathbf{N}}$ constitute zero mean white Gaussian noise in the space spanned by the columns of $\mathbf{P}_{\mathcal{I}_{k}}$, the pairwise error probability of the PIC-R considering the $k^{\text {th }}$ subsystem is given by

$$
\begin{aligned}
\operatorname{PEP}\left(\mathbf{X}_{1} \rightarrow \mathbf{X}_{2} \mid \mathbf{h}\right) & =Q\left(\frac{\sqrt{\rho}\left\|\mathbf{P}_{\mathcal{I}_{k}} \mathbf{G}_{\mathcal{I}_{k}} \Delta \mathbf{X}\right\|}{2}\right) \\
& \leq \frac{1}{2} \exp \left(\frac{-\rho\left\|\mathbf{P}_{\mathcal{I}_{k}} \mathbf{G}_{\mathcal{I}_{k}} \Delta \mathbf{X}\right\|^{2}}{4}\right)
\end{aligned}
$$

where $\mathbf{h}=\left[\operatorname{vec}\left(\mathbf{H}_{0}\right)^{T}, \operatorname{vec}\left(\mathbf{H}_{1}\right)^{T}, \ldots, \operatorname{vec}\left(\mathbf{H}_{P-1}\right)^{T}\right]^{T}, \mathbf{X}_{1}-$ $\mathbf{X}_{2}=\Delta \mathbf{X} \in \Delta \mathcal{C}^{\prime}=\left\{\mathbf{X}_{1}-\mathbf{X}_{2} \mid \mathbf{X}_{1} \neq \mathbf{X}_{2}, \mathbf{X}_{1}, \mathbf{X}_{2} \in \mathcal{C}^{\prime}\right\}$. For a given $\Delta \mathbf{X}$, we have $\left\|\mathbf{G}_{\mathcal{I}_{0}} \Delta \mathbf{X}\right\|^{2}=\left\|\mathbf{G}_{\mathcal{I}_{1}} \Delta \mathbf{X}\right\|^{2}=$ $\ldots=\left\|\mathbf{G}_{\mathcal{I}_{K-1}} \Delta \mathbf{X}\right\|^{2}$ owing to the block-Toeplitz structure of $\hat{\mathbf{H}}$. Furthermore, we have $\left\|\mathbf{G}_{\mathcal{I}_{0}} \Delta \mathbf{X}\right\|^{2}=\|\overline{\mathbf{H}} \Delta \mathbf{X}\|^{2}$, where $\overline{\mathbf{H}}=\left[\mathbf{H}_{0}^{T}, \mathbf{H}_{1}^{T}, \ldots, \mathbf{H}_{P-1}^{T}\right]^{T} \in \mathbb{C}^{P \times N_{t}}$. Since $\mathcal{C}^{\prime}$ is a fullrank STBC, we have $\operatorname{rank}(\Delta \mathbf{X})=N_{t}$ and the singular value decomposition of $\Delta \mathbf{X}$, which is given by $\mathbf{U} \mathbf{\Sigma} \mathbf{V}^{H}$, will have $N_{t}$ non-zero singular values. Thus, we have

$$
\begin{aligned}
\|\overline{\mathbf{H}} \Delta \mathbf{X}\|^{2} & =\|\overline{\mathbf{H}} \mathbf{U} \boldsymbol{\Sigma}\|^{2} \\
& =\operatorname{Tr}\left(\boldsymbol{\Sigma}^{T} \mathbf{U}^{H} \overline{\mathbf{H}}^{H} \overline{\mathbf{H}} \mathbf{U} \boldsymbol{\Sigma}\right) \\
& \geq \sigma_{N_{t}}^{2} \operatorname{Tr}\left(\overline{\mathbf{H}}^{H} \overline{\mathbf{H}}\right) \\
& =\sigma_{N_{t}}^{2}\|\mathbf{h}\|^{2}>0
\end{aligned}
$$

when $\mathbf{h} \neq \mathbf{0}$. Therefore, when $\mathbf{h} \neq \mathbf{0}$, we have $\mathbf{G}_{\mathcal{I}_{k}} \Delta \mathbf{X} \neq \mathbf{O}$ for $0 \leq k \leq K-1$ and any $\Delta \mathrm{X} \in \Delta \mathcal{C}^{\prime}$.

Assuming that $N_{r}(K+P-1) \geq N_{t} K^{4}, \hat{\mathbf{H}}$ will have a full column rank which ensures that

B1. if $v \in \operatorname{span}\left(\mathbf{G}_{\mathcal{I}_{k}}\right)$ then $v \notin \operatorname{span}\left(\mathbf{G}_{\mathcal{I}_{k}}^{c}\right)$.

Since $\mathbf{Q}_{\mathcal{I}_{k}}$ is a projection matrix onto the space spanned by the columns of $\mathbf{G}_{\mathcal{I}_{k}}^{c}$, we have $\mathbf{Q}_{\mathcal{I}_{k}} \mathbf{G}_{\mathcal{I}_{k}} \Delta \mathbf{X} \neq \mathbf{G}_{\mathcal{I}_{k}} \Delta \mathbf{X} \neq \mathbf{O}$, from which we obtain

$$
\begin{aligned}
\left(\mathbf{I}-\mathbf{Q}_{\mathcal{I}_{k}}\right) \mathbf{G}_{\mathcal{I}_{k}} \Delta \mathbf{X} \neq \mathbf{O}, \\
\mathbf{P}_{\mathcal{I}_{k}} \mathbf{G}_{\mathcal{I}_{k}} \Delta \mathbf{X} \neq \mathbf{O} .
\end{aligned}
$$

Therefore, we have $\left\|\mathbf{P}_{\mathcal{I}_{k}} \mathbf{G}_{\mathcal{I}_{k}} \Delta \mathbf{X}\right\|>0$ for $0 \leq k \leq K-1$. Denoting $\mathbf{P}_{\mathcal{I}_{k}}$ by $\mathbf{P}_{\mathcal{I}_{k}}(\mathbf{h})$ and $\mathbf{G}_{\mathcal{I}_{k}}$ by $\mathbf{G}_{\mathcal{I}_{k}}(\mathbf{h})$, it may be readily verified that $\mathbf{P}_{\mathcal{I}_{k}}\left(\frac{\mathbf{h}}{\|\mathbf{h}\|}\right)=\mathbf{P}_{\mathcal{I}_{k}}(\mathbf{h})$ and $\mathbf{G}_{\mathcal{I}_{k}}\left(\frac{\mathbf{h}}{\|\mathbf{h}\|}\right)=$ $\frac{1}{\|\mathbf{h}\|} \mathbf{G}_{\mathcal{I}_{k}}(\mathbf{h})$. Thus, restricting $\mathbf{h}$ to be from the unit sphere, we have $\left\|\mathbf{P}_{\mathcal{I}_{k}}\left(\frac{\mathbf{h}}{\|\mathbf{h}\|}\right) \mathbf{G}_{\mathcal{I}_{k}}\left(\frac{\mathbf{h}}{\|\mathbf{h}\|}\right) \Delta \mathbf{X}\right\|>0$. Since $\|\cdot\|$ is a uniformly continuous function and the unit sphere is a compact set, the co-domain of $\|\cdot\|$ will also be a compact set, and hence can be bounded [44], [45]. Thus, for any given $\Delta \mathbf{X}$ there exists a positive constant $c_{\Delta \mathbf{X}}$, such that $\left\|\mathbf{P}_{\mathcal{I}_{k}}\left(\frac{\mathbf{h}}{\|\mathbf{h}\|}\right) \mathbf{G}_{\mathcal{I}_{k}}\left(\frac{\mathbf{h}}{\|\mathbf{h}\|}\right) \Delta \mathbf{X}\right\|>c_{\Delta \mathbf{X}}$, which simplifies to

$$
\left\|\mathbf{P}_{\mathcal{I}_{k}} \mathbf{G}_{\mathcal{I}_{k}} \Delta \mathbf{X}\right\|>c_{\Delta \mathbf{X}}\|\mathbf{h}\| \text {. }
$$

Substituting (30) in (27), we arrive at

$$
\operatorname{PEP}\left(\mathbf{X}_{1} \rightarrow \mathbf{X}_{2} \mid \mathbf{h}\right) \leq \frac{1}{2} \exp \left(-\frac{\rho c_{\Delta \mathbf{X}}^{2}\|\mathbf{h}\|^{2}}{4}\right)
$$

Taking the expectation of (31) over $\mathbf{h}$ results in

$$
\operatorname{PEP}\left(\mathbf{X}_{1} \rightarrow \mathbf{X}_{2}\right) \leq \frac{1}{2}\left(\frac{4}{4+\rho c_{\Delta \mathbf{X}}^{2}}\right)^{N_{r} N_{t} P} .
$$

The union bound on the codeword error probability associated with each subsystem is given by

$$
P_{e}<\frac{1}{\left|\mathcal{C}^{\prime}\right|} \sum_{i=1}^{\left|\mathcal{C}^{\prime}\right|} \sum_{j=1}^{\left|\mathcal{C}^{\prime}\right|} \operatorname{PEP}\left(\mathbf{X}_{i} \rightarrow \mathbf{X}_{j}\right)
$$

It is clear from (32) and (33) that the PIC-R invoked in the ZP$\mathrm{SC}$ system in conjunction with the proposed grouping scheme of (13) achieves a diversity order of $N_{t} N_{r} P$. This concludes the proof.

\footnotetext{
${ }^{4}$ Note that this is a sufficient condition but not necessary. When $N_{r}(K+$ $P-1)<N_{t} K$, the condition B1 is still satisfied owing to the structure of $\hat{\mathbf{H}}$.
} 


\section{REFERENCES}

[1] P. Wolniansky, G. Foschini, G. Golden, and R. Valenzuela, "V-BLAST: an architecture for realizing very high data rates over the rich-scattering wireless channel," in Proc. 1998 International Symp. Signals, Syst., Electron., pp. 295-300.

[2] E. Viterbo and J. Boutros, "A universal lattice code decoder for fading channels," IEEE Trans. Inf. Theory, vol. 45, pp. 1639-1642, Jul. 1999.

[3] B. Hassibi and H. Vikalo, "On the sphere-decoding algorithm-I: expected complexity," IEEE Trans. Signal Process., vol. 53, no. 8, pp. 2806-2818, Aug. 2005.

[4] R. Mesleh, H. Haas, C. Ahn and S. Yun "Spatial modulation-a new low complexity spectral efficiency enhancing technique," in Proc. 2006 International Conf. Commun. Netw., pp. 1-5.

[5] R. Mesleh, H. Haas, S. Sinanovic, C. Ahn, and S. Yun "Spatial modulation," IEEE Trans. Veh. Technol., vol. 57, no. 4, pp. 2228-2242, July 2008.

[6] M. Di Renzo, H. Haas, and P. M. Grant, "Spatial modulation for multipleantenna wireless systems-a survey," IEEE Commun. Mag., vol. 49, no. 12, pp. 182-191, Dec. 2011.

[7] J. Jeganathan, A. Ghrayeb, and L. Szczecinski, "Spatial modulation: optimal detection and performance analysis," IEEE Commun. Lett., vol. 12, no. 8, pp. 545-547, Aug. 2008.

[8] R. Rajashekar and K.V.S. Hari, "Low complexity maximum likelihood detection in spatial modulation systems." Available: arXiv:1206.6190v1, June 2012

[9] S. Sugiura, C. Xu, S. X. Ng, and L. Hanzo, "Reduced-complexity coherent versus non-coherent QAM-aided space-time shift keying," IEEE Trans. Commun., vol. 59, no. 11, pp. 3090-3101, Nov. 2011.

[10] J. Wang, S. Jia, and J. Song, "Signal vector based detection scheme for spatial modulation," IEEE Commun. Lett., vol. 16, no. 1, pp. 19-21, Jan. 2012

[11] P. Yang, Y. Xiao, L. Li, Q. Tang, and S. Li, "An improved matched-filter based detection algorithm for space-time shift keying systems," IEEE Signal Process. Lett., vol. 19, no. 5, pp. 271-274, May 2012.

[12] Q. Tang, Y. Xiao, P. Yang, Q. Yu, and S. Li, "A new low-complexity near-ML detection algorithm for spatial modulation," IEEE Wireless Commun. Lett., vol. 2, no. 1, pp. 90-93, Feb. 2013.

[13] E. Basar, U. Aygolu, E. Panayirci, and H. V. Poor, "Performance of spatial modulation in the presence of channel estimation errors," IEEE Commun. Lett., vol. 16, no. 2, pp. 176-179, Feb. 2012.

[14] S. Sugiura and L. Hanzo, "Effects of channel estimation on spatial modulation," IEEE Signal Process. Lett., vol. 19, no. 12, pp. 805-808, Dec. 2012.

[15] M. Di Renzo and H. Haas, "Performance comparison of different spatial modulation schemes in correlated fading channels," in Proc. 2010 IEEE Int. Conf. Commun., pp. 1-6.

[16] E. Basar, U. Aygolu, E. Panayirci, and H. V. Poor, "Space-time block coding for spatial modulation," IEEE Trans. Commun., vol. 59, no. 3, pp 823-832, Mar. 2011.

[17] M. Di Renzo and H. Haas, "Transmit-diversity for spatial modulation (SM): towards the design of high-rate spatially-modulated space-time block codes," in Proc. 2011 IEEE Int. Conf. Commun.

[18] R. Rajashekar and K.V.S. Hari, "Modulation diversity for spatial modulation using complex interleaved orthogonal design," in Proc. 2012 IEEE TENCON, pp. 1-6.

[19] R. Y. Chang, S-J. Lin, and W-H. Chung, "Energy efficient transmission over space shift keying modulated MIMO channels," IEEE Trans. Commun., vol. 60, no. 10, pp. 2950-2959, Oct. 2012.

[20] M. Di Renzo and H. Haas, "Improving the performance of space shift keying (SSK) modulation via opportunistic power allocation," IEEE Commun. Lett., vol. 14, no. 6, pp. 500-502, June 2010.

[21] P. Yang, Y. Xiao, Y. Yu, and S. Li, "Adaptive spatial modulation for wireless MIMO transmission systems," IEEE Commun. Lett., vol. 15, no. 6, pp. 602-604, June 2011.

[22] P. Yang, Y. Xiao, L. Li, Q. Tang, Y. Yu, and S. Li, "Link adaptation for spatial modulation with limited feedback," IEEE Trans. Veh. Technol., vol. 61, no. 8, pp. 3808-3813, Oct. 2012.

[23] R. Rajashekar, K.V.S. Hari, and L. Hanzo, "Antenna selection in spatial modulation systems," IEEE Commun. Lett., vol. 17, no. 3, pp. 521-524, Mar. 2013.

[24] P. Som and A. Chockalingam, "Spatial modulation and space shift keying in single carrier communication," in Proc. 2012 IEEE PIMRC, pp. 1991-1996.

[25] M. I. Kadir, S. Sugiura, J .Zhang, S. Chen, and L. Hanzo, "OFDMA/SCFDMA aided space-time shift keying for dispersive multi-user scenarios," IEEE Trans. Veh. Technol., vol. 62, no. 1, pp. 408-414, Jan. 2013.
[26] L. P. Natarajan, P. K. Srinath, and B. S. Rajan, "Generalized distributive law for ML decoding of STBCs," in Proc. 2011 Inf. Theory Worksh.

[27] X. Guo and X.-G. Xia, "On full diversity space-time block codes with partial interference cancellation group decoding," IEEE Trans. Inf. Theory, vol. 55, pp. 4366-4385, Oct. 2009.

[28] X. Guo and X.-G. Xia, "Corrections to 'On full diversity space-time block codes with partial interference cancellation group decoding,', IEEE Trans. Inf. Theory, vol. 56, pp. 3635-3636, Jul. 2010.

[29] L. Shi, W. Zhang, and Z.-G. Xia, "High-rate and full-diversity spacetime block codes with low complexity partial interference cancellation group decoding," IEEE Trans. Commun., vol. 59, no. 5, pp. 1201-1207, May 2011.

[30] W. Zhang, T. Xu, and Z.-G. Xia "Two designs of space-time block codes achieving full diversity with partial interference cancellation group decoding," IEEE Trans. Inf. Theory, vol. 58, no. 2, pp. 747-764, Feb. 2012.

[31] J. Chun and T. Kailath, "Divide and conquer solutions of least squares problems for matrices with displacement structure," SIAM J. Matrix Anal. Appl., vol. 12, pp. 128-145, Jan. 1991.

[32] J. Chun and T. Kailath, "Generalized displacement structure for blocktoeplitz, toeplitz-block, and toeplitz-derived matrices," SIAM J. Matrix Anal. Appl., vol. 15, pp. 114-128, Jan. 1994.

[33] T. Kailath and A. Sayed, "Displacement structure: theory and applications," SIAM Review, vol. 37, no. 3, pp. 297-386, Sep. 1995.

[34] Z. Wang, X. Ma, and G. B. Giannakis, "Optimality of single-carrier zero-padded block transmissions," in Proc. 2002 IEEE Wireless Commun. Netw. Conf., pp. 660-664.

[35] Z. Wang, X. Ma, and G. B. Giannakis, "OFDM or single-carrier block transmissions?" IEEE Trans. Commun., vol. 52, no. 3, pp. 380-394, Mar. 2004.

[36] C. Tepedelenlioglu, "Low complexity linear equalizers with maximum multipath diversity for zero padded transmissions," in Proc. 2003 International Conf. on Acoustics, Speech and Sig. Process., vol. 4, pp. 636-639.

[37] B.S.M.R. Rao and K.V.S. Hari, "On the diversity and complexity of zero forcing receivers for MIMO zero padded systems," in Proc. 2010 Intern. Conf. on Signal Proc. and Commun.

[38] J. K. Zhang, J. Liu, and K. M. Wong, "Linear toeplitz space time block codes," in Proc. 2005 IEEE Int. Symp. Inf. Theory, pp. 1942-1946.

[39] Spatial channel model for Multiple Input Multiple Output (MIMO) simulations, 3GPP TR 25.996, version 11.0.0, Release 11. Available: http://www.3gpp.org/ftp/specs/html-INFO/25996.htm

[40] ETSI Normalization Committee, "Channel Models for HIPERLAN/2 in different indoor scenarios," Norme ETSI, document 3ER1085B, European Telecommunications Standards Institute, Sophia-Antipolis, Valbonne, France, 1998. Available: http://www.etsi.org.

[41] N. Al-Dhahir, "FIR channel-shortening equalizers for MIMO ISI channels," IEEE Trans. Commun., vol. 49, no. 2, pp. 213-218, Feb. 2001.

[42] D. Wubben and K.-D. Kammeyer, "Impulse shortening and equalization of frequency-selective MIMO channels with respect to layered space-time architectures," Signal Process., vol. 83, no. 8, pp. 1643-1659, 2003.

[43] D. Tse and P. Viswanath, Fundamentals of Wireless Communication. Cambridge University Press, 2005.

[44] W. Rudin, Principles of Mathematical Analysis. McGraw-Hill, 1976.

[45] G. F. Simmons, Topology and Modern Analysis. McGraw-Hill, 1963.

[46] M. R. Bhavani Shankar, "Design of linear precoded MIMO communication systems," Ph.D. thesis, Department of ECE, Indian Institute of Science, Apr. 2007. Available: http://hdl.handle.net/2005/558

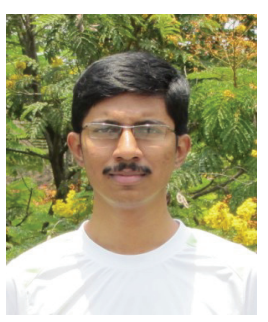

Rakshith Rajashekar received the B.E. degree in electrical communication engineering from Visvesvaraya Technological University, Karnataka, India, in 2007. He is currently a Ph.D. student in the Department of Electrical Communication Engineering, Indian Institute of Science (IISc), India. His research interests include wireless communication, space-time signal processing and coding. Before joining the Ph.D. program, he worked at Accord Software \& Systems, Bangalore, India, as a Systems Engineer from 2007 to 2009. 


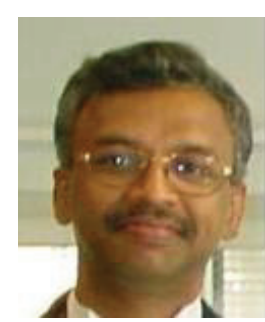

K.V.S. Hari (M'92-SM'97) received the B.E. (1983), M.Tech(1985) and $\mathrm{PhD}(1990)$ degrees from Osmania University, IIT Delhi, University of California at San Diego, respectively. Since 1992, he has been a Faculty Member at the Department of ECE, Indian Institute of Science (IISc), Bangalore, where he is currently a Professor and coordinates the activities of the Statistical Signal Processing Lab in the department. Currently, he is also an Affiliated Professor in the School of Electrical Engineering, KTH-Royal Institute of Technology, Stockholm, Sweden. He has been a visiting faculty member at Stanford University, KTH - Royal Institute of Technology and Helsinki University of Technology (now Aalto Univ). He also worked at DLRL, Hyderabad, and at the R\&D unit for Navigational Electronics, Osmania University. His research interests are in developing signal processing algorithms for MIMO wireless communication systems, sparse signal recovery problems, indoor positioning and DOA estimation. During his work at Stanford University, he worked on MIMO wireless channel modeling and is the coauthor of the WiMAX standard on wireless channel models for fixed-broadband wireless communication systems which proposed the Stanford University Interim (SUI) channel models. He is currently an Editor of the EURASIP's Journal on Signal Processing published by Elsevier. $\mathrm{He}$ is also an academic entrepreneur and is a cofounder of the company ESQUBE Communication Solutions, Bangalore.

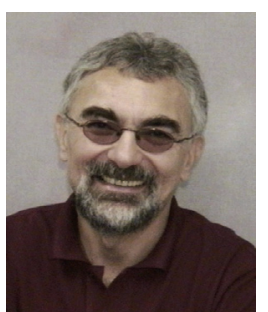

Lajos Hanzo (http://www-mobile.ecs.soton.ac.uk) FREng, FIEEE, FIET, Fellow of EURASIP, DSc received his degree in electronics in 1976 and his doctorate in 1983. In 2009 he was awarded the honorary doctorate "Doctor Honoris Causa" by the Technical University of Budapest. During his 35-year career in telecommunications he has held various research and academic posts in Hungary, Germany and the UK. Since 1986 he has been with the School of Electronics and Computer Science, University of Southampton, UK, where he holds the chair in telecommunications. He has successfully supervised $80 \mathrm{PhD}$ students, co-authored 20 John Wiley/IEEE Press books on mobile radio communications totalling in excess of 10000 pages, published 1300+ research entries at IEEE Xplore, acted both as TPC and General Chair of IEEE conferences, presented keynote lectures and has been awarded a number of distinctions. Currently he is directing a 100-strong academic research team, working on a range of research projects in the field of wireless multimedia communications sponsored by industry, the Engineering and Physical Sciences Research Council (EPSRC) UK, the European IST Programme and the Mobile Virtual Centre of Excellence (VCE), UK. He is an enthusiastic supporter of industrial and academic liaison and he offers a range of industrial courses. He is also a Governor of the IEEE VTS. During 2008 - 2012 he was the Editor-inChief of the IEEE Press and a Chaired Professor also at Tsinghua University, Beijing. His research is funded by the European Research Council's Senior Research Fellow Grant. For further information on research in progress and associated publications please refer to http://www-mobile.ecs.soton.ac.uk Lajos has $17000+$ citations. 